\title{
High-gradient low- $\beta$ accelerating structure using the first negative spatial harmonic of the fundamental mode
}

\author{
Sergey V. Kutsaev, ${ }^{1,{ }^{*}}$ Ronald Agustsson, ${ }^{1}$ Salime Boucher, ${ }^{1}$ Richard Fischer, ${ }^{2}$ Alex Murokh, ${ }^{1}$ \\ Brahim Mustapha, ${ }^{2}$ Alireza Nassiri, ${ }^{2}$ Peter N. Ostroumov, ${ }^{3,2}$ Alexander Plastun, ${ }^{3}$ \\ Evgeny Savin, ${ }^{1, \dagger}$ and Alexander Yu. Smirnov ${ }^{1}$ \\ ${ }^{1}$ RadiaBeam Technologies LLC, 1717 Stewart Street, Santa Monica, California 90404, USA \\ ${ }^{2}$ Argonne National Laboratory, 9700 South Cass Avenue, Lemont, Illinois 60439, USA \\ ${ }^{3}$ Facility for Rare Isotope Beams (FRIB), Michigan State University, 640 South Shaw Lane, \\ East Lansing, Michigan 48824, USA \\ (Received 23 May 2017; published 19 December 2017)
}

\begin{abstract}
The development of high-gradient accelerating structures for low- $\beta$ particles is the key for compact hadron linear accelerators. A particular example of such a machine is a hadron therapy linac, which is a promising alternative to cyclic machines, traditionally used for cancer treatment. Currently, the practical utilization of linear accelerators in radiation therapy is limited by the requirement to be under $50 \mathrm{~m}$ in length. A usable device for cancer therapy should produce $200-250 \mathrm{MeV}$ protons and/or $400-450 \mathrm{MeV} / \mathrm{u}$ carbon ions, which sets the requirement of having $35 \mathrm{MV} / \mathrm{m}$ average "real-estate gradient" or gradient per unit of actual accelerator length, including different accelerating sections, focusing elements and beam transport lines, and at least $50 \mathrm{MV} / \mathrm{m}$ accelerating gradients in the high-energy section of the linac. Such high accelerating gradients for ion linacs have recently become feasible for operations at S-band frequencies. However, the reasonable application of traditional S-band structures is practically limited to $\beta=\mathrm{v} / \mathrm{c}>0.4$. However, the simulations show that for lower phase velocities, these structures have either high surface fields $(>200 \mathrm{MV} / \mathrm{m})$ or low shunt impedances $(<35 \mathrm{M} \Omega / \mathrm{m})$. At the same time, a significant $(\sim 10 \%)$ reduction in the linac length can be achieved by using the $50 \mathrm{MV} / \mathrm{m}$ structures starting from $\beta \sim 0.3$. To address this issue, we have designed a novel radio frequency structure where the beam is synchronous with the higher spatial harmonic of the electromagnetic field. In this paper, we discuss the principles of this approach, the related beam dynamics and especially the electromagnetic and thermomechanical designs of this novel structure. Besides the application to ion therapy, the technology described in this paper can be applied to future high gradient normal conducting ion linacs and high energy physics machines, such as a compact hadron collider. This approach preserves linac compactness in settings with limited space availability.
\end{abstract}

DOI: 10.1103/PhysRevAccelBeams.20.120401

\section{INTRODUCTION}

Radiation therapy is a large segment of clinical oncology, where the treatment is performed by delivering ionizing radiation beams directly to the tumor. Existing radiation therapy machines can use beams of $\mathrm{x}$ rays or hadrons (i.e., protons or positive ions) for treatment, and although about $60 \%$ of all cancer patients undergo some form of radiotherapy, only about $1 \%$ of all radiotherapy patients receive treatment by high energy

\footnotetext{
Corresponding author. kutsaev@radiabeam.com

"Present address: National Research Nuclear University "MEPhI," Russia.

Published by the American Physical Society under the terms of the Creative Commons Attribution 4.0 International license. Further distribution of this work must maintain attribution to the author(s) and the published article's title, journal citation, and DOI.
}

ions [1]. Yet, hadron therapy provides significantly better localization of the dose to the tumor and much lower dose to healthy tissues, compared to traditional $\mathrm{x}$-ray therapy, due to the characteristic "Bragg peak" depth-dose distribution in tissues. As a result, in some estimates, as much as $15 \%$ of patients can benefit from hadron therapy options [2].

Unfortunately, the high cost of treatments using both proton and carbon beams is the limiting factor preventing hadron therapy from becoming the standard of care for a wider range of cancers. A usable device for cancer therapy needs to produce $200-250 \mathrm{MeV}$ protons or/and up to $400-450 \mathrm{MeV} / \mathrm{u}$ carbon ions [3]. Both cyclotrons and synchrotrons that are currently used for this purpose are expensive and bulky in size with large magnets and have significant limitations with fast energy adjustment and power efficiency [4]. A cyclotron/synchrotron proton therapy center costs more than $\$ 100$ million [5], and carbon therapy centers cost a factor of 2 more. In comparison, a standard photon therapy linac costs about $\$ 3$ million, and 

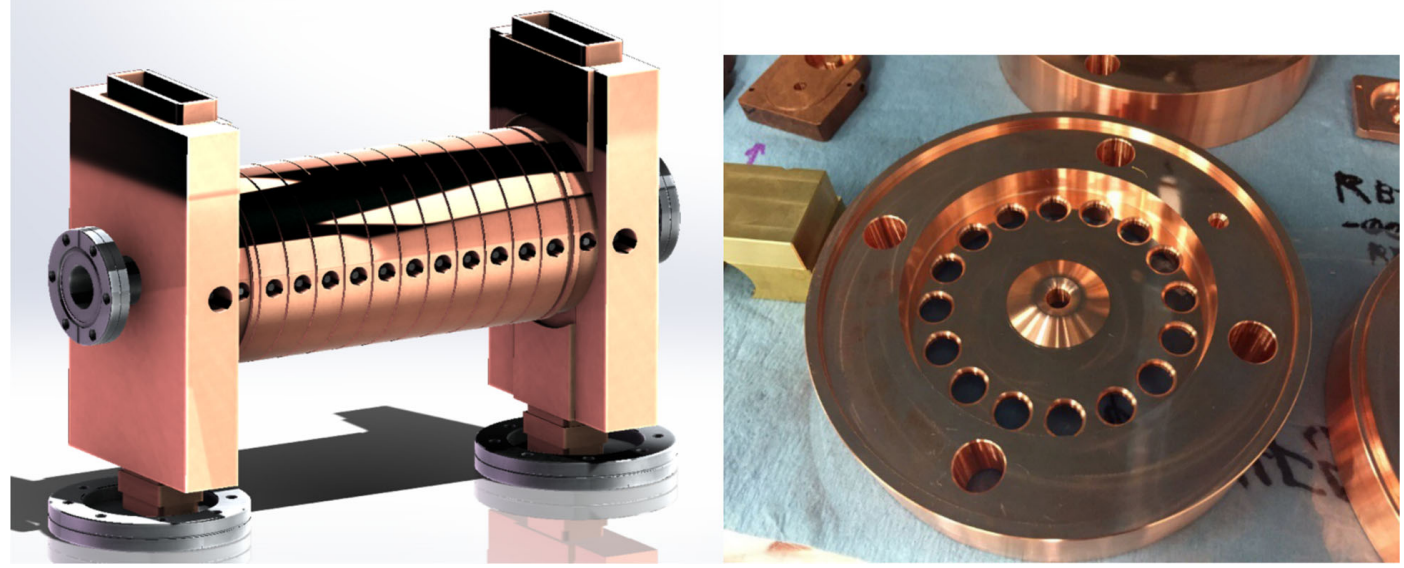

FIG. 1. Left: 3D layout rendering of the 15 -cell $\beta=0.3$ negative harmonic structure. Right: Fabricated cell prototype of the $\beta=0.3$ negative harmonic structure.

even with civil construction costs, a brand new conventional radiotherapy clinic costs less than $\$ 10$ million [6].

Within hadron therapy, proton therapy is the most common [7], although studies indicate that carbon ion therapy offers additional advantages [8], and a combined proton and carbon therapy could achieve the most precise dose delivery to the tumor [9], while sparing healthy tissues. This is why having a system that can provide both proton and carbon beams with rapid energy adjustment is highly desirable, but that is beyond the capability of cyclotrons and synchrotrons presently used in the field.

There are several projects worldwide $[10,11]$ pursuing the development of compact hadron therapy linacs. The concept of these linacs is based on the use of high-gradient accelerating structures (HGS) after the linac front end. Among the proposed structures, only TULIP's [12] backward-traveling wave structure (BTW) [13] comes close to providing the required rf parameters. However, these BTW cells are designed for $\beta=0.38$. Proton acceleration from $10 \mathrm{MeV}(\beta=0.15)$ to $70 \mathrm{MeV}(\beta=0.38)$ is performed in a 4 meters long side-coupled drift-tube linac structure (SCDTL). For ${ }^{12} \mathrm{C}^{6+}$ ions with $\mathrm{q} / \mathrm{A}$ ratio of $1 / 2$, this length will double. By starting the acceleration of carbon ions at high gradient from $45 \mathrm{MeV} / \mathrm{u}(\beta=0.3)$, it is possible to reduce the length of the SCDTL by half $(4 \mathrm{~m})$ and replace the other half with three HGS sections with a total length of $\sim 1 \mathrm{~m}$. This approach is a key feature of the Advanced Compact Carbon Ion Linac (ACCIL) recently designed at Argonne National Laboratory [14].

The direct scaling of BTW cells design from $\beta=0.38$ to $\beta=0.3$ beam velocities leads to intolerable peak fields $(>220 \mathrm{MV} / \mathrm{m})$, well above the demonstrated levels of $192 \mathrm{MV} / \mathrm{m}$ for a single structure [15]. For multicell rf guns the achieved peak electric field limits are lower: $\sim 160 \mathrm{MV} / \mathrm{m}$ [16]. This happens due to the very close nose arrangement, or to dramatically lower power efficiencies if the noses around the aperture are removed, which lowers the shunt impedance.
To work around this issue, we designed a cavity where the beam is synchronous with the higher spatial harmonic of the electromagnetic field in the periodic disk-loaded structure [17]. The structure shown in Fig. 1 is designed to operate at the -1 st harmonic, capable of producing $50 \mathrm{MV} / \mathrm{m}$ gradient at peak field levels $\sim 20 \%$ lower than in TULIP and thus lower rf breakdown rate than the $\beta=$ 0.3 BTW with no noses operating at the fundamental harmonic. The comparison of the parameters of TULIP, LIBO [18], and CABOTO [19] structures against the proposed structure is presented in Table I. We have performed the thermostructural analysis of the designed 15-cell accelerating section, including waveguide couplers to ensure its stable operation at the design gradients with the required duty factors. We have also fabricated and measured one prototype cell of the structure to ensure that the required tolerances can be achieved.

The presented design offers some attractive features.

(i) The structure can provide accelerating gradients of up to $50 \mathrm{MV} / \mathrm{m}$ for particles with $\beta=0.3$, which will exceed the gradient of SCDTL linacs with typical values of about $15 \mathrm{MV} / \mathrm{m}[21,22]$.

(ii) The novel negative harmonics approach allows the use of noses near the aperture, increasing the power efficiency of the structure by $50 \%$ compared to a traditional fundamental harmonic structure with no noses.

(iii) The optimized elliptical shape of the noses keeps the peak electric fields below $160 \mathrm{MV} / \mathrm{m}$.

(iv) It is possible to vary magnetic coupling hole dimensions in the disk-loaded structure to allow a constant gradient at the required $50 \mathrm{MV} / \mathrm{m}$ level along the whole length of the multicell structure without tapering the aperture radius.

(v) The designed structure is scalable and can be used either for lower- or higher- $\beta$ ions.

In the following sections, we will discuss in detail each aspect of the high-gradient low- $\beta$ structure based on acceleration with the first negative spatial harmonic. 
TABLE I. Comparison of developed high gradient structures for hadron therapy linacs.

\begin{tabular}{lcccc}
\hline \hline Structure & ACCIL & TULIP [13] & LIBO [20] & CABOTO \\
\hline Ion types & $\mathrm{p}^{+}$and ${ }^{12} \mathrm{C}^{6+}$ & $\mathrm{p}^{+}$ & $\mathrm{p}^{+}$ & ${ }^{12} \mathrm{C}^{6+}$ \\
Minimum $\beta$ & 0.30 & 0.38 & 0.25 & 0.65 \\
Frequency, MHz & 2856 & 2998.5 & 2998.5 & 2998.5 \\
Structure type & $\mathrm{BTW}$ & $\mathrm{BTW}$ & $\mathrm{SCL}$ & SCL \\
Spatial harmonic & $-1 \mathrm{st}$ & Fundamental & Fundamental & Fundamental \\
Accelerating gradient, $\mathrm{MV} / \mathrm{m}$ & 50 & 50 & 10 & $\sim 15$ \\
Section length, cm & 27.6 & 19.0 & 20.0 & 48.8 \\
Number of cells & 15 & 12 & 16 & 15 \\
Shunt impedance, $\mathrm{M} \Omega / \mathrm{m}$ & 32 & 52 & 29 & 100 \\
Peak electric field, $\mathrm{MV} / \mathrm{m}$ & 160 & 146 & $\ldots$ & $\ldots$ \\
Modified Poynting vector, $\mathrm{MW} / \mathrm{mm}^{2}$ & 1.3 & 0.8 & 3.0 & $\ldots$ \\
Beam pulse width, $\mu \mathrm{s}$ & 1.0 & 2.5 & 1.5 & 3.0 \\
Filling time, $\mu \mathrm{s}$ & 0.35 & 0.23 & 200 & 1.5 \\
Repetition rate, $\mathrm{pps}$ & 120 & 20.6 & 3.0 & 400 \\
Input rf power, MW & 42.7 & & 4.5 \\
\hline \hline
\end{tabular}

\section{REQUIREMENTS FOR THE $\beta=0.3$ SECTION PARAMETERS}

To define the requirements for the proposed negative harmonic HGS, we used the conceptual design of the ACCIL [14,23] as a starting point. First, we have performed the detailed beam dynamics study of the accelerating section for proton and carbon ions with $\beta=0.3$ and defined the requirements and basic parameters for the rf accelerating structure to be used in this section.

In principle, the S-band structure of the main part of ACCIL can be either standing wave (SW) or traveling wave (TW) mode structures. Each tank consists of several identical cells, the number varying per tank and depending on the phase velocity normalized to the speed of light (later in the text referred to simply as phase velocity) $\beta$, and must provide an accelerating gradient of $50 \mathrm{MV} / \mathrm{m}$ to ensure a linac footprint of no more than $50 \mathrm{~m}$. Such high accelerating gradients required for the ACCIL linac are feasible due to the operation at high frequency $(2856 \mathrm{MHz})$ and very low duty cycle $(<0.06 \%)$ with very short beam pulses $(<0.5 \mu \mathrm{s})$.

We have chosen the S-band frequency as a compromise between the availability of high power rf sources, the known peak electric fields demonstrated at certain frequencies $(\sim 160 \mathrm{MV} / \mathrm{m}$ for S-band [15], and $\sim 250 \mathrm{MV} / \mathrm{m}$ for $\mathrm{X}$-band [24,25], and the references therein), and the higher effective shunt impedance $\left(Z \cdot T^{2}\right)$. If all the dimensions are scaled with frequency, the shunt impedance $Z$ scales as $f^{1 / 2}$. However, if we fix the aperture size, the shunt impedance and the transit-time factor (TTF) $T$ will become dependent on the frequency. The concept of a transit-time factor is valid both to standing and traveling wave structures. In realistic TW structures the field amplitude and phase distributions are different from the ideal as shown in Fig. 2. Thus, the TTF can be described as follows [26]:

$$
T=\frac{\int_{-L / 2}^{L / 2}\left(E_{A} \cos k_{z} z+E_{S} \sin k_{z} z\right) d z}{\int_{-L / 2}^{L / 2} \sqrt{E_{A}^{2}+E_{S}^{2}} d z} .
$$

Here, $E_{A}$ and $E_{S}$ are the solutions of Maxwell's equations representing the longitudinal component standing waves, which are respectively antisymmetrical and symmetrical with respect to the middle plane of the cell. According to [17], for the structures where phase advance is not a multiple of $\pi$, the shunt impedance of TW structure is linearly related to that of SW structure as

$$
Z(S W) \approx \frac{8}{\pi^{2}} Z(T W)
$$

Thus, we can use the concept of TTF and the formula for the simplest case of the pillbox cavity [27] to estimate the TTF, which for the fixed aperture radius $a$, scales with the operating wavelength $\lambda$ as

$$
T \sim \frac{\sin (g / \beta \lambda)}{g / \beta \lambda} \frac{1}{I_{0}\left(\frac{2 \pi}{\gamma \beta \lambda} a\right)} .
$$

Here $g$ stands for accelerating gap length, $I_{0}$ is the modified Bessel function, and $\gamma$ is the relativistic mass factor. The plot of the effective shunt impedance of the conventional pillbox cavity with the fixed size of the aperture as a function of phase velocity, computed numerically for different frequencies and shown in Fig. 3 demonstrates that for $\beta$ range from 0.3 to 0.4 , S-band provides the highest power efficiency compared to L- and C-bands. Then, by iterative $\mathrm{rf}$ and beam dynamics calculations, described below, we found the minimum aperture of $6 \mathrm{~mm}$ as a compromise between beam acceptance and shunt impedance. The optimal 

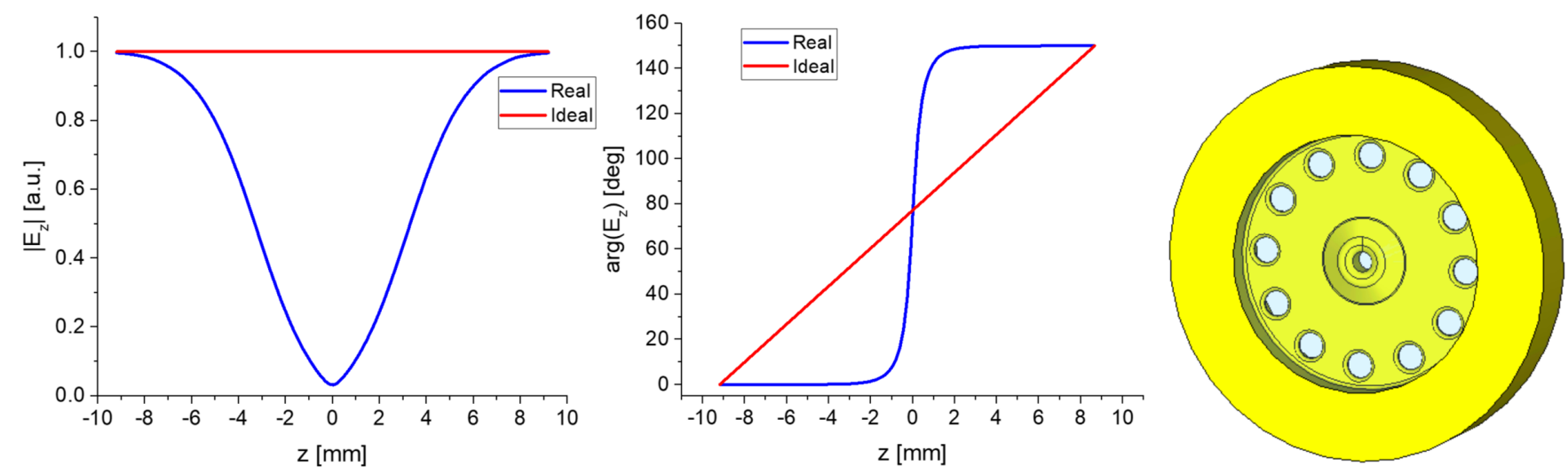

FIG. 2. Realistic (blue) and ideal (center) amplitude (left) and phase (center) distributions of traveling wave field in the middle of the $\beta=0.425 \pi / 6$ magnetic-coupled disk-loaded waveguide cell (right) as simulated in CST MicrowAVE STUDIO.

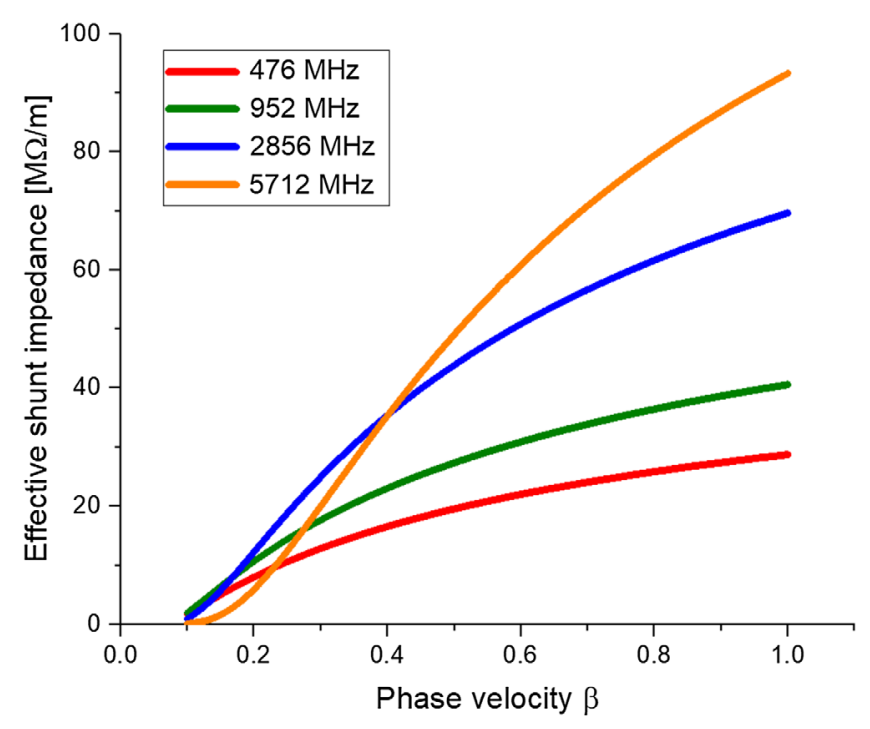

FIG. 3. Effective shunt impedance of a $\pi$-mode pillbox cavity as a function of phase velocity for different frequencies. Beam apertures are $6 \mathrm{~mm}$.

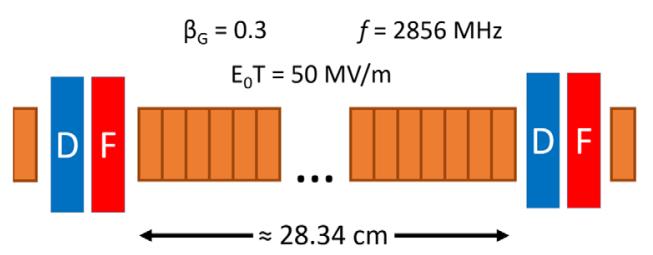

FIG. 4. Schematics of the accelerator scheme with the focusing lattice.

frequency remains the same for different aperture sizes in the $\beta$ range of interest.

For the beam dynamics simulation inside the $\beta=0.3$ section, modeled as single cells, we used the TRACK code [28]. The current ACCIL design assumes a series of multicell accelerating sections with focusing quadrupole doublets in between, as shown in Fig. 4. Each

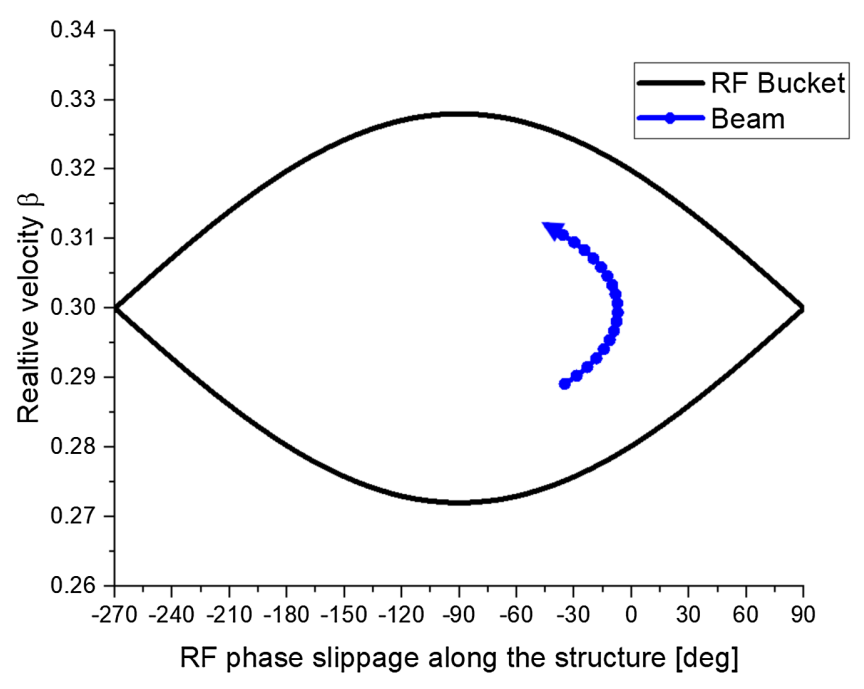

FIG. 5. Phase slippage of the beam center in a $28.5 \mathrm{~cm}$ long $\beta=0.3$ section. The dots represent different cells.

tank consists of cells with constant phase velocity and length. Thus, the rf phase of the beam center slips around the reference phase $\varphi_{r}$, which is defined as an average $\mathrm{rf}$ phase of the beam center in the whole tank (Fig. 5). The phase slippage is a parameter that limits the accelerating section length. The simulations demonstrate that since the beam velocity kick is relatively high, the phase slippage in a $30-\mathrm{cm} \beta=0.3$ section is about $50^{\circ}-$ $60^{\circ}$ from the reference phase $\varphi_{r}=-20^{\circ}$, which was chosen as a compromise between energy gain rate and stability area.

The transverse phase advance of a particle in a doublet lattice is defined in the smooth approximation as [29]

$$
\sigma_{T}^{2}=\left[\frac{q G l \sqrt{L D}}{m c \gamma \beta}\right]^{2}+\frac{\pi q E_{0} T \sin (\varphi) P^{2}}{m c^{2} \lambda(\gamma \beta)^{3}}
$$




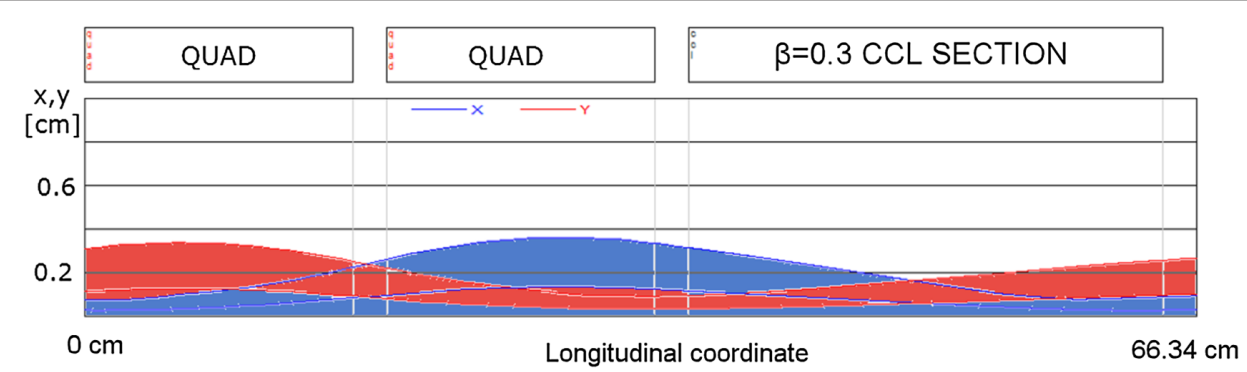

FIG. 6. The results of beam dynamics simulations in $\beta=0.350 \mathrm{MV} / \mathrm{m}$ section produced by TRACK. Beam envelope development along the section ( $\mathrm{x}$-blue; $\mathrm{y}$-red).

where $q$ and $m$ are charge and mass of the beam particle, $G$ is gradient of the magnetic quadrupole, $l$ is length of the quadrupole, $P$ is focusing period, $\gamma$ is relativistic factor of the beam particle, $L$ and $D$ are the distances between the centers of adjacent quadrupoles, and $P=L+D$ is the focusing period of the doublet lattice. The smooth approximation is applicable in our case since it is valid for low $(<60-70$ degrees $)$ phase advances of transverse oscillations. Although the doublet lattice allows having longer accelerating sections than a FODO lattice for the same phase advance and rf defocusing strength, it requires higher gradient integrals $G \cdot l$ for the quadrupoles than the FODO. As shown in Fig. 6, TRACK simulations with $140 \mathrm{~T} / \mathrm{m}$ quadrupoles demonstrated that to ensure transverse focusing, the aperture diameter of the $\beta=0.3$ section must be no less than $6 \mathrm{~mm}$, and the section length to be less than $28.5 \mathrm{~cm}$. For the input parameters of the ${ }^{12} \mathrm{C}^{6+}$ ion beam for simulations, we assumed the following: (i) transverse normalized $90 \%$ emittance of $0.35 \pi \mathrm{mm} \cdot \mathrm{mrad}$, which is similar to the value from commercially available ECR ion sources [30]; (ii) normalized longitudinal 90\% emittance of $\sim 0.85 \mathrm{keV} / \mathrm{u} \cdot \mathrm{ns}$, bunch length of $\sim 8 \mathrm{deg}$, and energy spread of $\sim 2 \%$ were calculated in TRACK [23].

The detailed description of the ACCIL linac and its simulations will be presented elsewhere. The final requirements for the structure are summarized in Table II.

TABLE II. The requirements for the ACCIL $\beta=0.3$ section.

\begin{tabular}{lc}
\hline \hline Parameter & Value \\
\hline Operating frequency & $2856 \mathrm{MHz}$ \\
Phase velocity, $\beta$ & 0.3 \\
Accelerating gradient (constant along & $50 \mathrm{MV} / \mathrm{m}$ \\
$\quad$ the section) & \\
Length of the section & $28.34 \mathrm{~cm}$ \\
Aperture diameter & $\geq 6 \mathrm{~mm}$ \\
Beam pulse width & $1.0 \mu \mathrm{s}$ \\
Filling time & $<0.5 \mu \mathrm{s}$ \\
Repetition rate & $120 \mathrm{~Hz}$ \\
\hline \hline
\end{tabular}

\section{NEGATIVE HARMONIC ACCELERATING STRUCTURE}

\section{A. High gradient structures}

There are several known criteria for rf breakdown limits that we have used for the EM design.

(i) Peak surface electric fields of $250 \mathrm{MV} / \mathrm{m}$ at $11-$ $12 \mathrm{GHz}$ and $\sim 160 \mathrm{MV} / \mathrm{m}$ for S-band have been demonstrated in rf guns and side-coupled linacs [15,24].

(ii) Peak surface magnetic field that causes pulse heating, which can damage the structure if the peak temperature rise is higher than $50^{\circ} \mathrm{C}$ [31].

(iii) There are also new theories of a unified criterion, such as a modified Poynting vector $(\langle\mathrm{Sc}\rangle)$ that may impact the gradient. However, there is not much experimental data for S-band structures. The predicted limit for the structure with the parameters specified in Table II was scaled from the numbers published in [13] to $1 \mu \mathrm{s}$ pulse length and found to be $2.3 \mathrm{MW} / \mathrm{mm}^{2}$.

For the design of the $50 \mathrm{MV} / \mathrm{m}, \beta=0.3$ structure, we decided to keep all three parameters below these limits.

Typically, the $\pi$-mode coupled cavity linac (CCL) structure is used for high-gradient structures because of its low peak field to accelerating gradient ratio [32]. Although CCL-type accelerating structures demonstrate excellent performance when designed for particles with high $\beta$, its rf shunt impedance degrades dramatically for structures with $\beta<0.7$ (see Fig. 3). Also, when designing the multicell structure, it is necessary to take into account the neighbor mode separation, which is relatively small for the $\pi$-mode. For a 28.5 -cm $\pi$-mode section, 18 cells are required, which will result in sub-MHz mode separation, making it extremely sensitive to mechanical and other errors [33]. This problem can be solved either by developing a standing wave (SW) structure, operating in $\pi / 2$-mode or by switching to the traveling wave (TW) operation regime. In our previous work [34], we have excluded the side-coupled linac (SCL), on-axis coupled biperiodic structure (BPS), and disk-and-washer (DAW) structure options for the $\beta=0.43$ structure for having about twice higher filling time, modified Poynting vector, and pulsed heating values, while not offering 


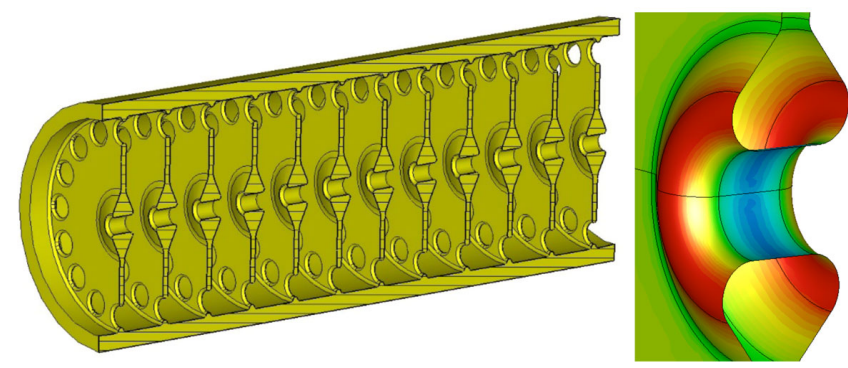

FIG. 7. BTW structure model (left) and electric field distribution in the nose part for the $50 \mathrm{MV} / \mathrm{m}$ gradient (right). Red color corresponds to $200 \mathrm{MV} / \mathrm{m}$.

any improvement in peak electric field and shunt impedance values. Thus, for $\beta=0.3$, these structures are even less feasible.

For TW structures, such as the disk-loaded waveguide, to compensate the field attenuation due to power losses, the iris diameter must be reduced along the waveguide. This solution is not practical due to the restriction of $6 \mathrm{~mm}$ aperture diameter, set by the transverse beam oscillation, while the increase of the aperture is not efficient from the shunt impedance point of view. In this case, the coupling can be provided by magnetic fields via coupling holes in the iris. This allows for increasing the coupling coefficient, leaving the aperture radius constant and thus not reducing the shunt impedance of the cell. The magnetic coupling makes the dispersion negative, so the phase and group velocities of the positive spatial harmonic have different signs, and the rf power propagates oppositely to the beam direction, in the so-called backward traveling wave (BTW) regime [35]. As a reference design, we used the CERN $50 \mathrm{MV} / \mathrm{m}, \quad \beta=0.38,2856-\mathrm{MHz}, 5 \pi / 6$ magnetically coupled structure that was designed for the TULIP proton therapy accelerator [13]. Figure 7 shows the $\beta=$ 0.43 BTW section designed for ACCIL.

Despite its attractiveness, the TULIP design has a significant drawback that prevents it from being adapted to $\beta=0.3$. The design peak electric field for $\beta=0.38$ is $200 \mathrm{MV} / \mathrm{m}$ for $50 \mathrm{MV} / \mathrm{m}$ accelerating gradient, well above the $160 \mathrm{MV} / \mathrm{m}$ and breakdown rate of $10^{-6}$ at $1 \mu$ s pulse length, which we consider to be the demonstrated reliable RFBD limit. These peak field values are located at the nose tips of the structure (see Fig. 7). By reducing the phase velocity to $\beta=0.3$, the cell length $L=\frac{\beta \lambda \theta}{2 \pi}$ and the distance between noses becomes $25 \%$ shorter, which makes the peak electric fields unacceptably high: $>1 \mathrm{GV} / \mathrm{m}$ for shunt impedance of $30 \mathrm{M} \Omega / \mathrm{m}$ and accelerating gap of $5 \mathrm{~mm}$. Since the maximum available gap length is $8.5 \mathrm{~mm}$ (no noses), no further optimization was done for this type of structure. In this case, to bring the fields down to $160 \mathrm{MV} / \mathrm{m}$ level, the noses must be removed from the cell geometry. Such change significantly reduces the concentration of the

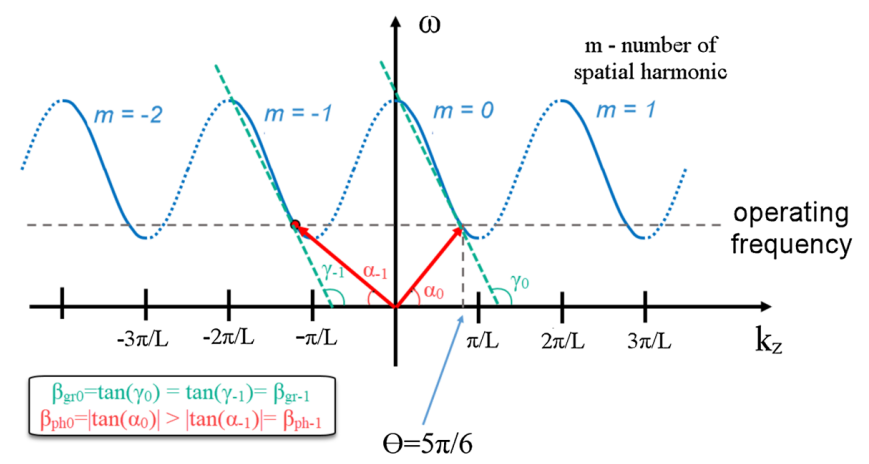

FIG. 8. The dispersion curve of disk-loaded periodic waveguide structures with a magnetic coupling ( $n=0$ curve corresponds to the fundamental harmonic, $n=-1$ curve corresponds to the first negative harmonic).

electric field near the aperture and thus reduces the effective shunt impedance by $\sim 40 \%$ as calculated for the case of $\beta=0.43$ [34].

To work around this issue, we came up with a design of the cavity where the beam is synchronous not with the fundamental spatial harmonic but with the higher spatial harmonic of the electromagnetic field in the periodic structure. Periodic structures have an infinite number of spatial harmonics (see Fig. 8). These harmonics have the same frequency but different spatial field distribution. An accelerating structure can be designed not only for the fundamental harmonic $m=0$ but also for the $m=-1$ harmonic which will make the accelerating period longer [36]. In structures with longer periods, it is now possible to implement noses that will increase the shunt impedance without a significant increase in the peak fields. In addition, by going from $\beta=0.4$ to 0.3 , it is challenging to reduce the iris thickness proportionally which makes it even more attractive to use longer cells, operating at the first negative harmonic. One important difference between operating magnetically coupled disk loaded waveguide structure at the $m=0$ and $m=-1$ modes is the fact that, for $m=0$ it is backward wave mode which means that the group and phase velocities have opposite directions, whereas for $m=-1$ it is forward wave mode with group and phase velocities going in the same direction.

\section{B. rf design}

The rf design optimization of the accelerating cell included the choice of the optimal phase advance per cell, optimization of the coupling holes and noses, and the preliminary structural stability considerations. The aim is to maximize the shunt impedance of the structure while keeping the peak electric fields below $160 \mathrm{MV} / \mathrm{m}$, the pulsed heating gradient below $50 \mathrm{~K}$, and the modified Poynting vector module below $2.3 \mathrm{MW} / \mathrm{mm}^{2}$. The final parameters were then compared with those of a traditional 


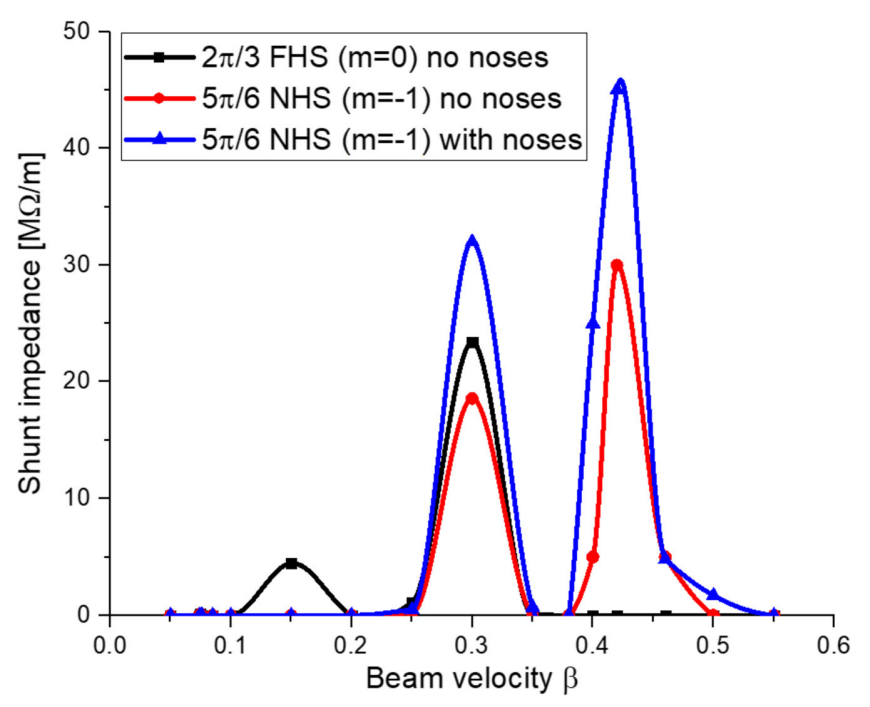

FIG. 9. Shunt impedance of the 20-cells BTW structures with different geometry and designed to operate at different spatial harmonics, as a function of beam velocity. The geometry of FHS and NHS cells with no noses is similar except for the cell length.

BTW structures with $\beta=0.3$ operating at the fundamental harmonic.

First, we have studied how the shunt impedance of the fundamental harmonic BTW cell with fixed aperture radius of $3 \mathrm{~mm}$, coupling hole diameter of $8 \mathrm{~mm}$, and iris halfthickness $1.5 \mathrm{~mm}$, is affected by the phase advance. From these studies, we found that the highest shunt impedance is achieved for $\theta=2 \pi / 3$, which agrees well with previous work [37], so these parameters will be used as a reference for comparison with those of the negative-harmonic structure.

For the structure where the beam is synchronous with the spatial harmonic of order $\mathrm{m}$, the accelerating cell length is calculated as

$$
L=\frac{\beta_{m} \lambda|\theta+2 \pi m|}{2 \pi} .
$$

For $m=-1, L=\frac{\beta \lambda(2 \pi-\theta)}{2 \pi}$, the cell is $\frac{2 \pi}{\theta}-1$ times longer than the one operating at the fundamental harmonic. Conversely, for the same cell length, the phase velocity of the -1 st harmonic will be $\frac{2 \pi}{\theta}-1$ times lower than the phase velocity of the fundamental harmonic. For example, according to this formula for $5 \pi / 6$ mode $\beta_{-1}=0.3$ and

$$
\beta_{0}=0.3 \cdot\left(\frac{2 \pi}{\frac{5 \pi}{6}}-1\right)=0.42 \text {. }
$$

Similarly, for $2 \pi / 3$ structure with $\beta_{0}=0.3$,

$$
\beta_{-1}=0.3 /\left(\frac{2 \pi}{\frac{2 \pi}{3}}-1\right)=0.15 \text {. }
$$

We have created a 20-cell model with periodic boundary conditions. In this model, we have calculated the shunt impedance of the structure for beams traveling with various $\beta$ 's. In these simulations performed in CST MiCROWAVE STUDIO, we have considered boundary conditions with both positive and negative phase advance, corresponding to forward and backward waves, so two peaks are present. The consistency of the 20-cells model ensures the fact that for a beam that is not synchronous with any harmonic, the voltage gain would be zero. Figure 9 demonstrates that in the simulated structures, the beam is synchronous with both the fundamental harmonic and the first negative harmonic, which are $\beta=0.3$ and $\beta=0.15$ in case of fundamental harmonic structure (FHS at $2 \pi / 3$ ), black curve, and $\beta=$ 0.42 and $\beta=0.3$ in case of the first negative harmonic structure (NHS at $5 \pi / 6$ ), red and blue curves, respectively. This demonstrates the proof-of-principle acceleration of the particles in the structure operating at the higher harmonics.
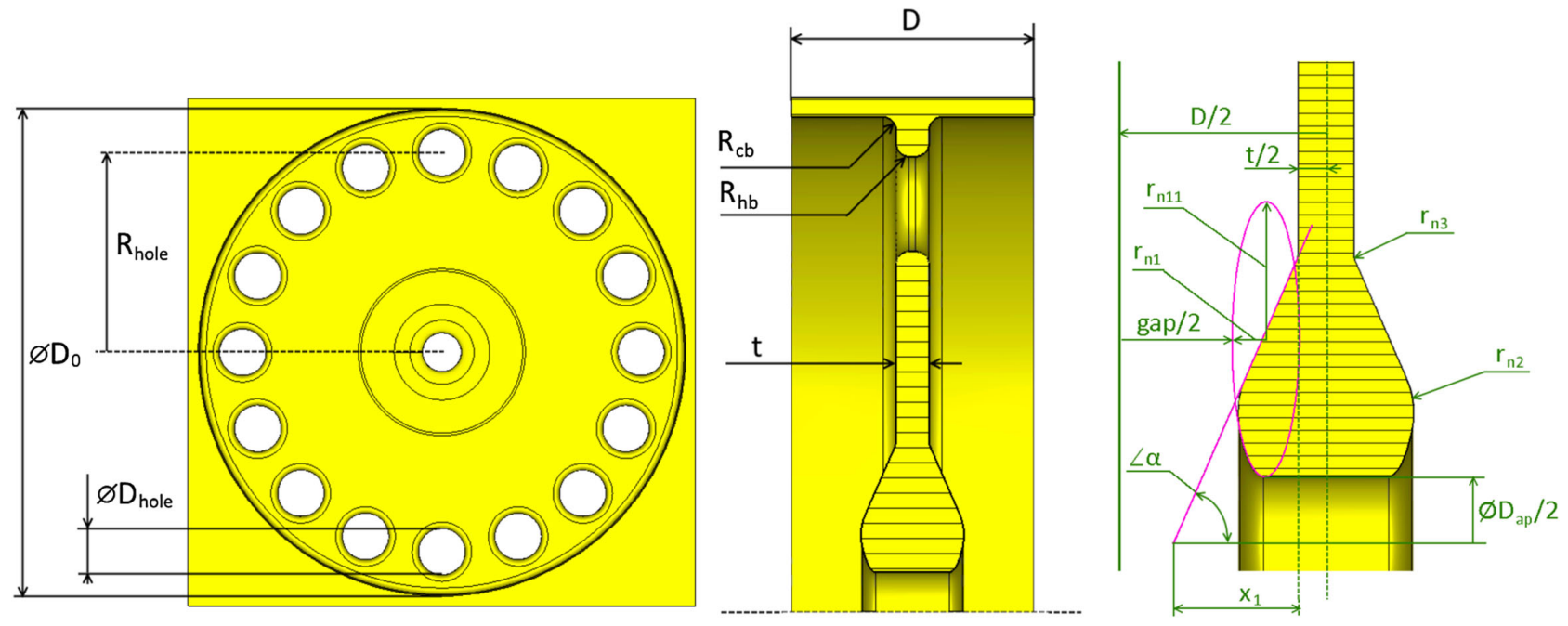

FIG. 10. Profile of the $\beta=0.3$ NHS cells with the optimized parameters. 


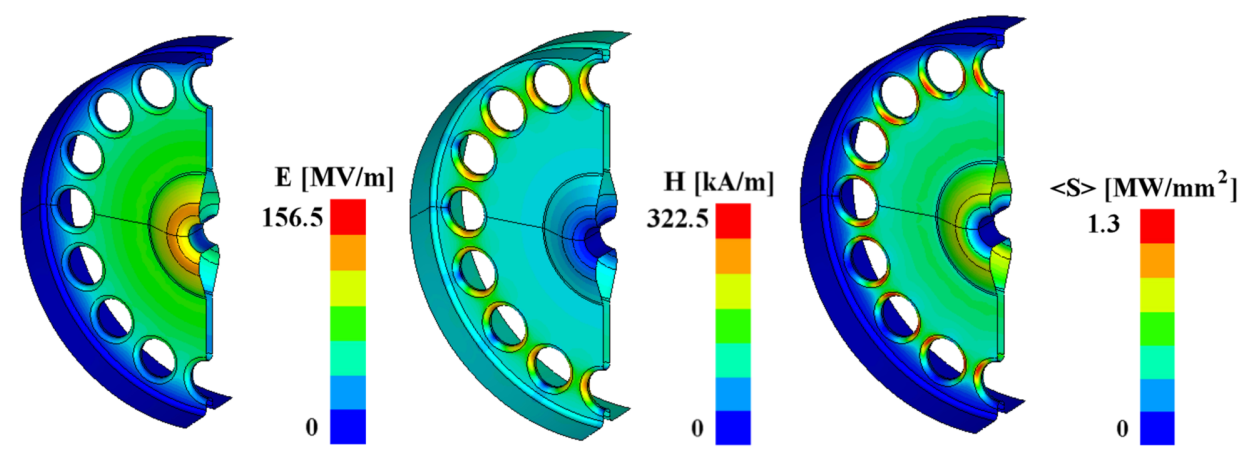

FIG. 11. Electric (left), magnetic (center) and modified Poynting vector (right) field maps in the optimized $\beta=0.3$ NHS cell.

As seen in Fig. 9, the amplitudes of higher harmonics reduce dramatically, so the resulting shunt impedance of the $2 \pi / 3$ FHS (black curve) is $23 \%$ times higher than the shunt impedance of the $5 \pi / 6$ NHS without noses (red curve). To compensate the reduced energy gain due to the smaller amplitude, we have introduced aperture noses similar to those shown in Fig. 7 for the NHS, which will concentrate the electric field near the beam axis and improve the shunt impedance (blue curve).

We have designed an elliptical profile for the nose as shown in Fig. 7 to minimize the surface electric field. The nose profile is formed by a cylindrical surface and the intersection of an ellipse with a cone. Magnetic coupling between cells is performed with 16 holes in the iris with uniform angular spacing. Then, we optimized the accelerating gap, the nose ellipse profile, and the angle to get the highest shunt impedance while targeting the ratio of peak electric field to the accelerating gradient $\mathrm{E}_{\max } / \mathrm{E}_{\mathrm{acc}}$ to be $\leq 3.2$, which corresponds to $160 \mathrm{MV} / \mathrm{m}$ maximum surface electric field. The optimized shape and its dimensions are shown in Fig. 10. Figure 11 presents the distribution of the electric field strength in this cell. Since, the coupling holes arrangement does not have any dipole or quadruple asymmetry, the dipole and quadrupole field distortion would be negligible and much weaker than the rf defocusing.

We have done the studies of the optimal phase advance per cell in the NHS, similar to the studies done for the FHS. However, in this case, we also considered the peak electric field in addition to shunt impedance. Unlike the FHS, in negative harmonic BTW, the maximum shunt impedance and lower peak electric field are achieved for phase advances closer to $\pi$. Since the group velocity of the $\pi$-mode is zero, we have chosen to operate at $5 \pi / 6$ mode as shown in Fig. 12. We have also performed preliminary thermostructural simulations in CST MuLTIPhysics STUdio to verify the structural stability of the cells and to define the minimal iris thickness for which the mechanical von Mises stresses are below $70 \mathrm{MPa}$, which corresponds to the yield stress of annealed copper [38].

The comparison of the optimized FHS and NHS cells with and without noses (FHS cannot fit the noses as
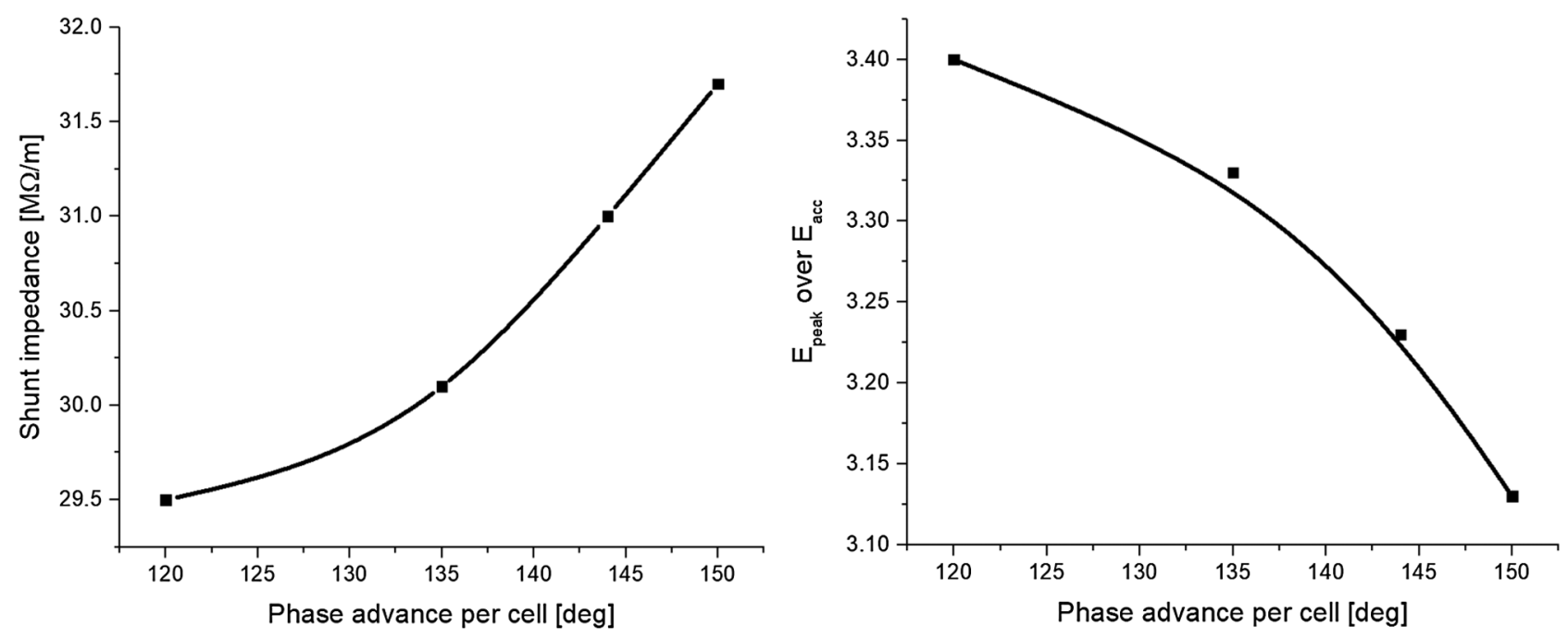

FIG. 12. Shunt impedance (left) and peak field normalized to the accelerating gradient (right) of the NHS structure with noses as a function of designed operation mode. 
TABLE III. The comparison of rf parameters for $\beta=0.3$ FHS and NHS end cells.

\begin{tabular}{lccc}
\hline \hline Structure type & FHS & NHS & \\
\hline Harmonic & 0 & -1 & -1 \\
Cell length, mm & 10.5 & 18.375 & 18.375 \\
Mode of operation & $2 \pi / 3$ & $5 \pi / 6$ & $5 \pi / 6$ \\
Noses & No & No & Yes \\
Iris thickness, mm & 2 & 3 & 2.5 \\
Gap length, mm & 8.5 & 15.375 & 10.725 \\
Number of cells & 26 & 15 & 15 \\
Shunt impedance, M $\Omega / m$ & 22.9 & 18.58 & 31.7 \\
Q-factor & 4960 & 7680 & 8065 \\
Group velocity (out), \% of c & -0.49 & 0.19 & 0.26 \\
Fundamental harmonic velocity $\beta$ & 0.3 & 0.42 & 0.42 \\
Particle velocity $\beta$ & 0.3 & 0.3 & 0.3 \\
Accelerating gradient, MV/m & 50 & 50 & 50 \\
Peak E-field, MV/m & 92.5 & 130 & 156.5 \\
Modified Poynting vector, & 1.4 & 2.0 & 1.3 \\
$\quad$ MW/mm ${ }^{2}$ & & & \\
Pulsed heating @ 1 $\mu$ s, K & 24 & 33.5 & 28.2 \\
Temperature gradient, ${ }^{\circ} \mathrm{C}$ & 39.2 & 21.2 & 15.6 \\
Peak mechanical stresses, MPa & 57 & 75 & 59.6 \\
\hline \hline
\end{tabular}

discussed above) are summarized in Table III. The NHS structure with noses provides $44 \%$ higher shunt impedance than the FHS while preserving the low pulsed heating and modified Poynting vector value; it also has a peak surface electric field lower than the $160 \mathrm{MV} / \mathrm{m}$ threshold and $\sim 30 \%$ lower than the $\beta=0.38$ BTW structure built by CERN [13]. Given these results, we have decided to proceed with the NHS structure with noses as the optimal design of the high gradient structure for $\beta=0.3$ particles.

We performed beam simulations of a round ${ }^{12} \mathrm{C}^{6+}$ beam with $3.6 \mathrm{~mm}$ diameter with zero length injected in the rf phase of -20 degrees to verify that the particle interacts with the negative harmonic and gains enough energy. We used CST PARTICle StUdio since it can correctly model the periodical structures with the phase advance boundary conditions without cylindrical symmetry (due to coupling holes). CST simulations show that the energy gain in such a case is $83.6 \mathrm{MeV}$ (voltage gain $=13.93 \mathrm{MV}$ for ${ }^{12} \mathrm{C}^{6+}$ ) in $27.57 \mathrm{~cm}$ (see Fig. 13), which corresponds to $50 \mathrm{MV} / \mathrm{m}$ gradient. We noticed that the beam dynamics simulation did not show any interaction with the fundamental harmonic of the electromagnetic field.

\section{Coupling and tuning}

Further steps of the structure design include all aspects of the accelerating section. To maintain an average $50 \mathrm{MV} / \mathrm{m}$ gradient along the structure, design of a constant gradient between cells is required. The group velocity must be varied along the structure to maintain a constant accelerating gradient. In BTW structures, the coupling between cells, which defines the group velocity of the structure, is provided by the magnetic field via coupling holes in the iris. This allows increasing the coupling coefficient while keeping the aperture radius small, and thus not reducing the shunt impedance of the cell.

As mentioned earlier, for BTW structure the $m=-1$ harmonic will operate in the forward wave regime. In practical terms, if for $m=0 \mathrm{rf}$ power and proton beam are injected from opposite ends of the structure, for $m=-1$ they are injected from the same side.

The rf design of the full accelerating section in CST Microwave Studio included: (i) design of the constant gradient section; (ii) calculation of group velocity modulation; (iii) electromagnetic tuning of the accelerating cells; (iv) design of input and output couplers.

Figure 14 shows the design of input and output rf power couplers and the distribution of the rf electric field amplitude along the tuned accelerating structure, corresponding to $14 \mathrm{MV}$ voltage gain $(50 \mathrm{MV} / \mathrm{m})$. The group velocity was adjusted along the structure according to Fig. 15(a) to keep the accelerating gradient constant. Figure 15(b) demonstrates that the shunt impedance variation is small and can be considered constant. The couplers were tuned to keep the reflection from the input coupler below $-30 \mathrm{~dB}$ as shown in Fig. 16. $50 \%$ of the input power is going to the load.
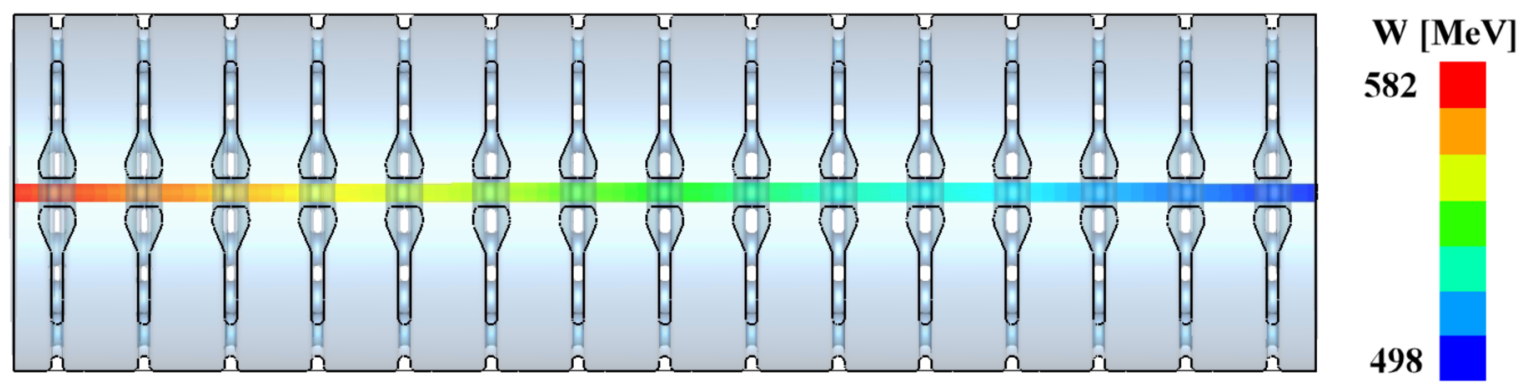

FIG. 13. Energy gain (in color) of $45 \mathrm{MeV} / \mathrm{u} 12 \mathrm{C} 6+$ beam at -20 degrees synchronous phase in 15 cells section of $\beta=0.3 \mathrm{NHS}$ as simulated in CST PARTICLE STUDiO. 

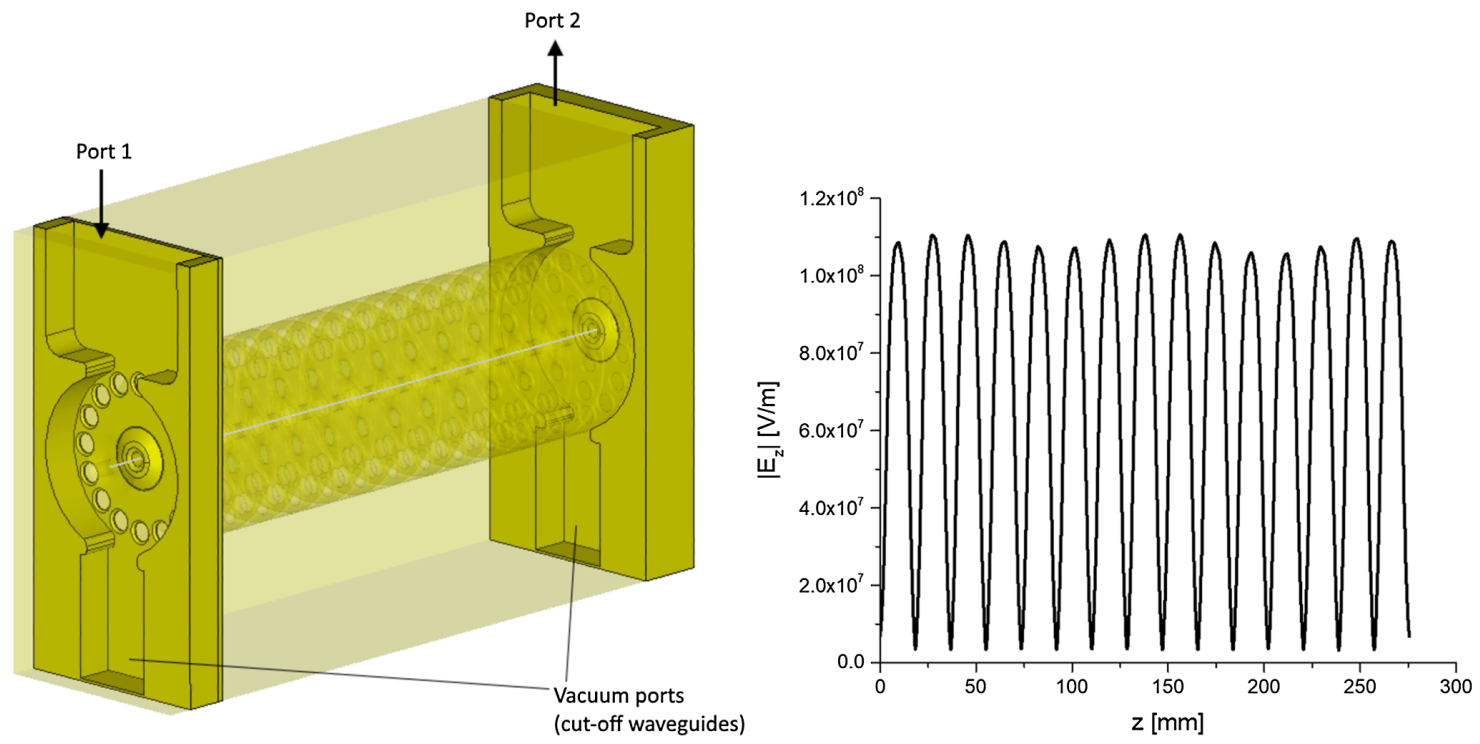

FIG. 14. Left: The design of input and output rf power couplers. Right: Profile of the complex amplitude of the electric field along the NHS constant gradient section with $42.7 \mathrm{MW}$ input power.
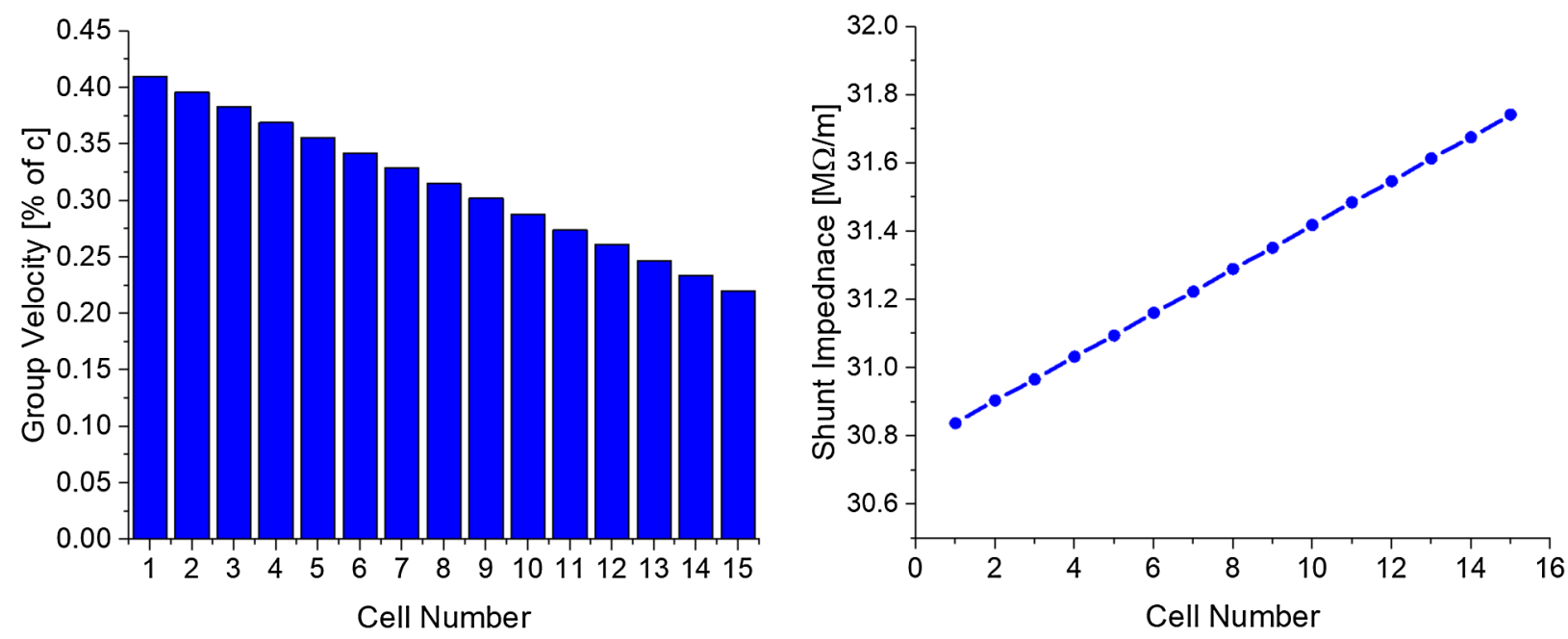

FIG. 15. Group velocity (left) and shunt impedance (right) variation in the cells along the structure.

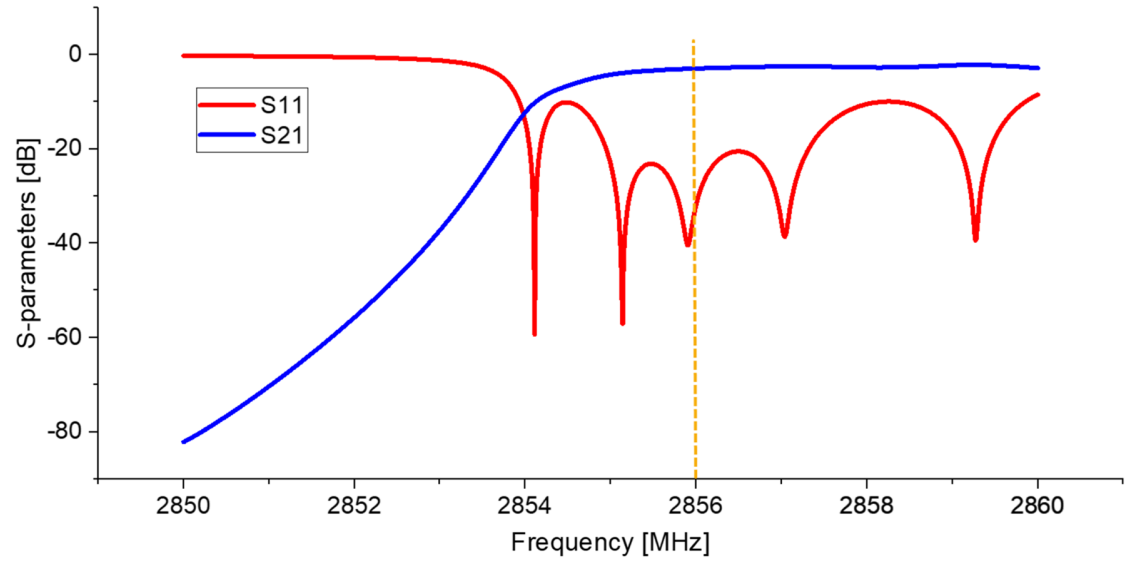

FIG. 16. Reflection (S11, red) and transmission (S21, green) characteristics of the NHS 15-cells structure with couplers. 


\section{CONCEPTUAL ENGINEERING DESIGN}

The conceptual mechanical design of the structure was then generated (shown in Fig. 1) and thermomechanical analysis completed. The engineering design incorporates realistic features required for vacuum, bonding, and tuning. To handle vacuum, as this is a TW structure, we will utilize field symmetrizing dummy ports at both input and output couplers to serve as vacuum pumping connections to maximize vacuum conductance. These ports are designed with a brazed-on cap to which a ConFlat flange can be welded to ensure the flange knife edge does not thermal cycle during brazing. Tuning will be performed through bidirectional push-pull-style tuners brazed on every cell.

\section{A. Thermal analysis}

The cavity was analyzed with the finite-element program ANSYS. Both steady-state and transient heat transfer analyses with thermal flux loads were conducted to determine the cavity temperature. The proof-of-principle analysis is based on the following assumptions: (i) materials are homogeneous and isotropic; (ii) elastic/linear plastic material response; (iii) thermal material properties are constant in time and temperature; (iv) structural material properties are constant in time but temperature dependent; (v) no creep or swelling effects; (vi) no thermal resistance at brazed joints; (vii) deformations are small; (viii) residual stresses are ignored.

The thermal analyses assumed the realistic heat flux generated by the magnetic field; this was calculated in CST MicrowAVE STUDIO and used as a thermal load in ANSYs. The flux was pulsed at $120 \mathrm{~Hz}$, with a pulse width of $1.0 \mu \mathrm{s}$, which results in a total power of $2500 \mathrm{~W}$ for the full model. The film coefficient used for the cooling water at the cavity's outer diameter (OD) was determined by first considering the flow rate required to keep the increase in coolant temperature below $5^{\circ} \mathrm{C}$. This value is commonly used in designing cooling systems in injection moulds [39]. The flow rate required to dissipate $P=2500 \mathrm{~W}$ with a $\Delta T=5{ }^{\circ} \mathrm{C}$ is

$$
Q=\frac{P}{\rho C_{p} \Delta T}=1.21 \cdot \frac{10^{-4} m^{3}}{s}=1.9 \mathrm{gpm} .
$$

The minimum film coefficient was found using

$$
h=\frac{P}{A \Delta T}
$$

with A taken as the area of the OD of a cell times 12 or $0.07 \mathrm{~m}^{2}, \quad h=6912 \mathrm{~W} /\left(\mathrm{m}^{2}{ }^{\circ} \mathrm{C}\right)$. The width of each cell is $18.375 \mathrm{~mm}$, so a helical cooling channel with an $18-\mathrm{mm}^{2}$ cross section was used to estimate the Nusselt number. Based on [40], the Nusselt number for a flow of 1.9 GPM is 643.9. This results in a film coefficient of $23,000 \mathrm{~W} /\left(\mathrm{m}^{2}{ }^{\circ} \mathrm{C}\right)$. Note that this is well above the minimum value calculated above. The flow rate equates to a water velocity of $1.22 \mathrm{ft} / \mathrm{s}(0.372 \mathrm{~m} / \mathrm{s})$, which is well below the $15 \mathrm{ft} / \mathrm{s}$ limits commonly applied to this type of equipment. This established the cooling capacity that could be easily provided with a simple water jacket. The analysis was run with convection values below this limit, and $10,000 \mathrm{~W} / \mathrm{m}^{2}-{ }^{\circ} \mathrm{C}$ was chosen as it produced acceptable temperatures without exhausting the available cooling capacity.

Boundary conditions for the thermal analysis consisted of a convection on the OD of the center 12 cells of $10,000 \mathrm{~W} / \mathrm{m}^{2}{ }^{\circ} \mathrm{C}$, convection in the cooling channels of $1000 \mathrm{~W} / \mathrm{m}^{2}-{ }^{\circ} \mathrm{C}$, both due to $40^{\circ} \mathrm{C}$ cooling water, and convection of $2 \mathrm{~W} / \mathrm{m}^{2}{ }^{\circ} \mathrm{C}$ due to natural convection with ambient air on the exterior surfaces. Results of the steadystate thermal analysis of the structure are shown in Fig. 17. The temperature distribution along the structure is nonuniform due to the differences in the thermal conductivity of each cell because of different coupling hole dimensions. This may cause field distortions in the operational regime. To deal with this effect, we plan to calculate the frequency shift of each cell due to the heating and tune the structure appropriately according to these frequency shift corrections. Figure 18 shows the transient temperature change in large scale and with rf pulse resolution, calculated with
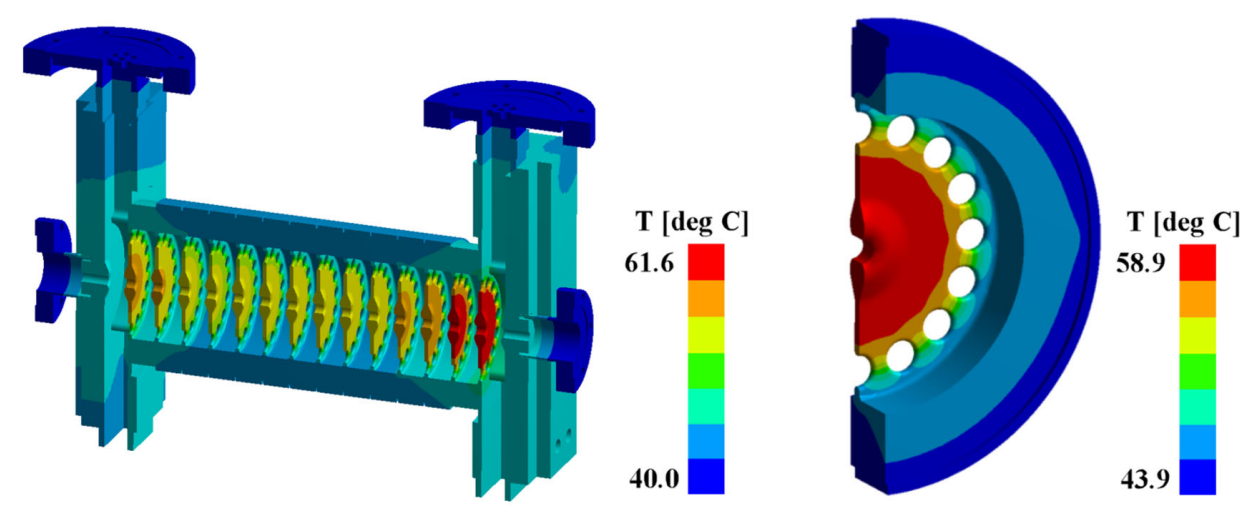

FIG. 17. Temperature map of the full structure (left) and cell \#12 (right) in the steady-state regime. 

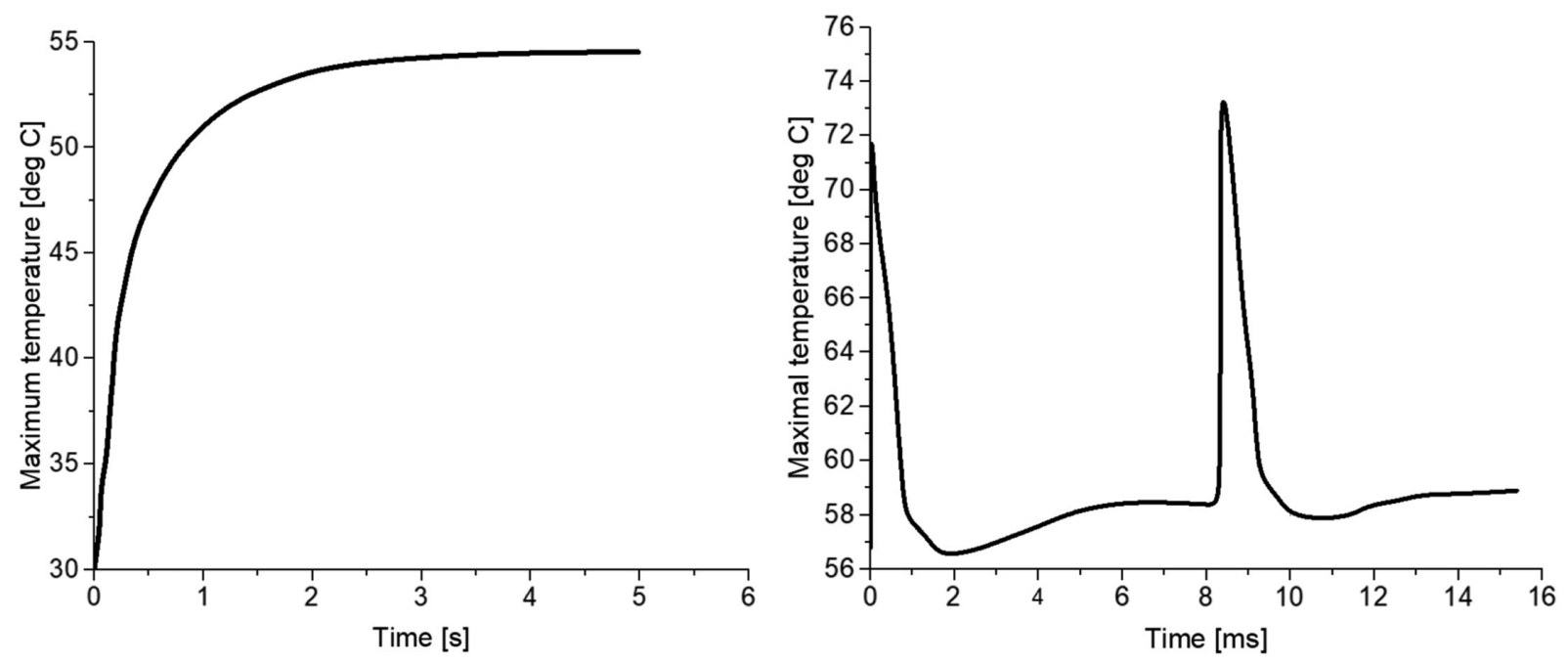

FIG. 18. Transient temperature analysis results. Left: in large time scale. Right: with rf pulse resolution. The results are taken at the point with the maximal temperature.
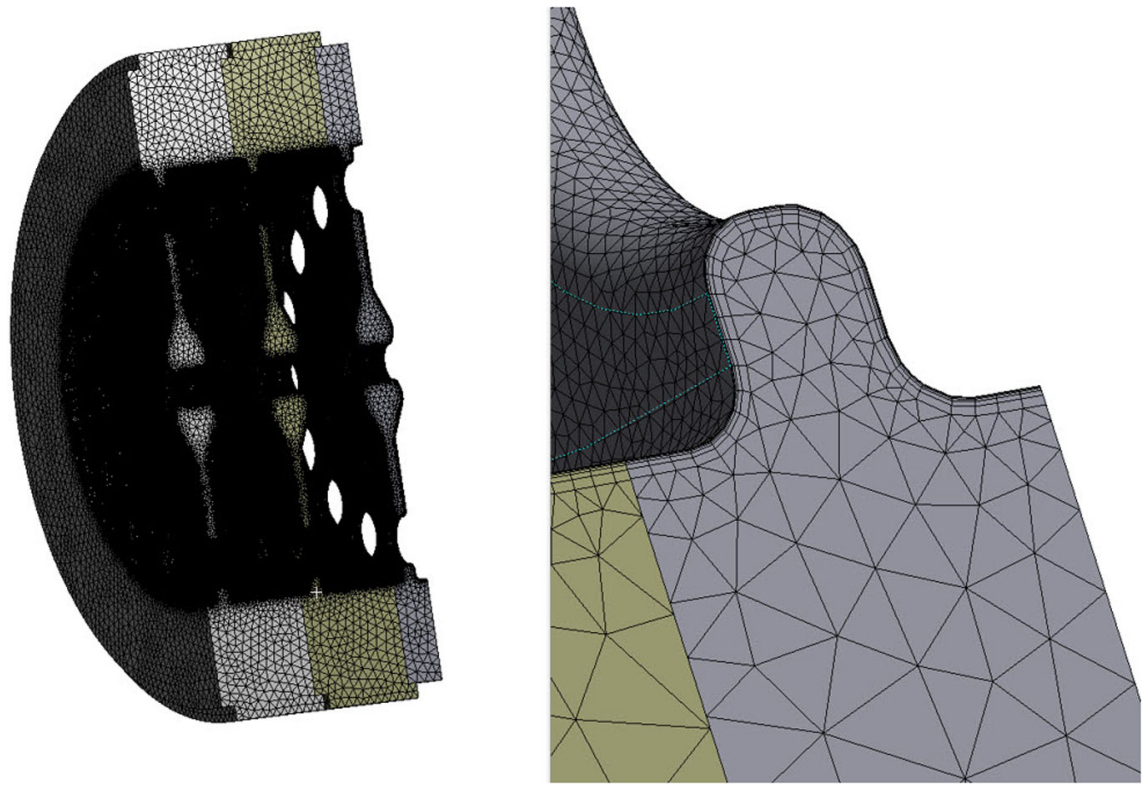

FIG. 19. ANSYS mesh for transient thermal analysis model.

$0.1 \mu$ s time step. Since the latter simulations are very time consuming and required a specialized mesh that was suitable for applying a surface flux with a short time step, shown in Fig. 19, we have simulated only two rf pulses. The local pulsed peak heating is $\sim 17$ degrees above the steady-state temperature during the pulse, which is lower than the analytical estimations (Table III) and well below the recommended limit of $50 \mathrm{~K}$ [31].

\section{B. Structural analysis}

Loading for the structural analysis consisted of vacuum, physical constraints, and temperatures from the thermal analyses. All material data used in the structural analysis
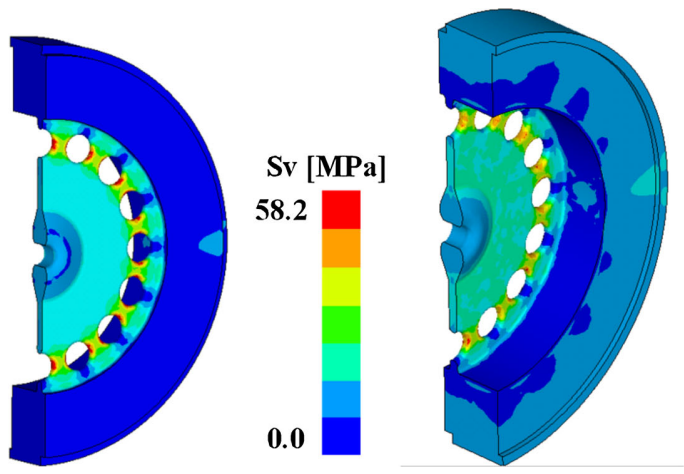

$\varepsilon[$

$2.9 \cdot 10^{-4}$

FIG. 20. Von Mises stress (left) and the first principal total strain (right) maps of the cell \#12 at the end of the fifth pulse. 

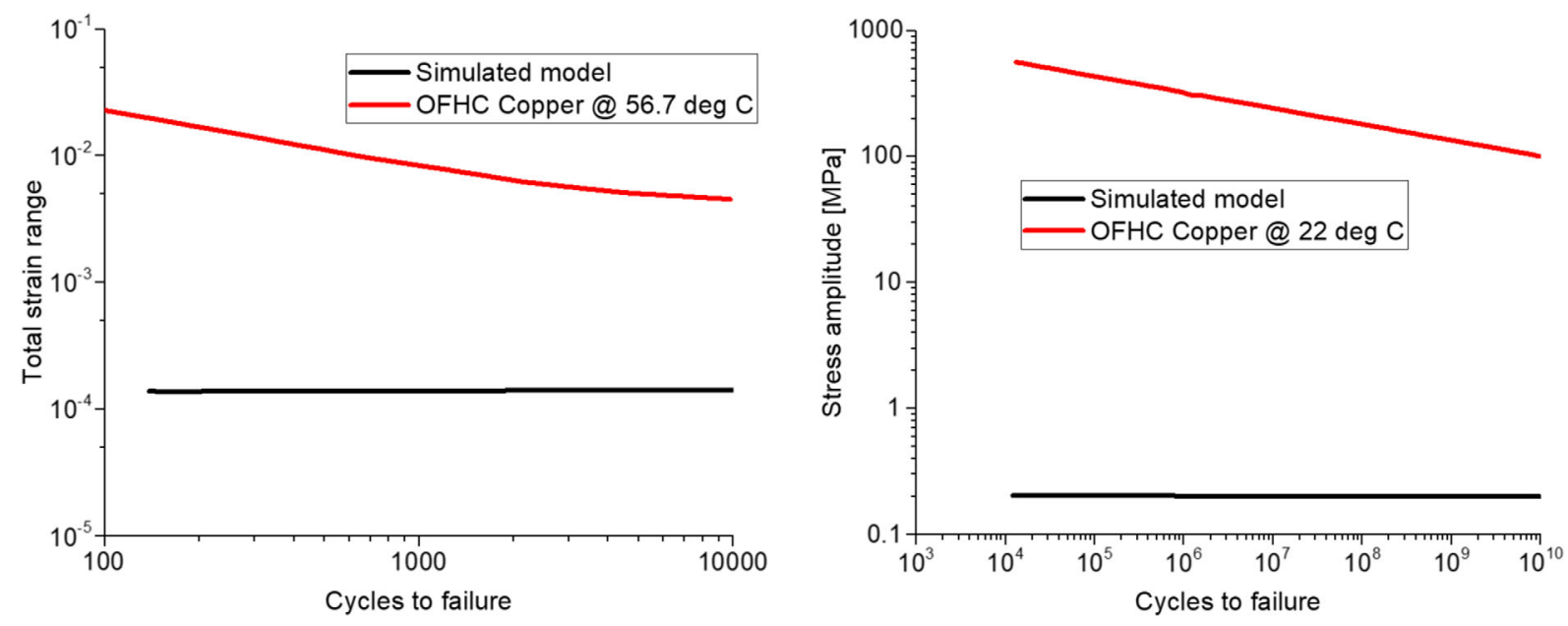

FIG. 21. Left, low cycle fatigue and, right, high cycle fatigue of copper. The black line is the value for the designed structure.

was taken in the annealed state. This included Young's modulus, yield strength, the stress-strain curve used for the BISO material model, and the low and high cycle fatigue data taken from [41,42]. The physical constraints were chosen to approximate a stress-free state. The heat load consisted of the temperature distribution from the steadystate analysis, with temperatures from the transient analysis superimposed on the target cell and the two adjacent cells. Two load cases consisting of the temperature at the end of the last pulse and the temperature at the end of the last cycle were run to determine the mean and alternating stresses. The structural analysis results are presented in Fig. 20.

At $70^{\circ} \mathrm{C}$, copper yield occurs at about $51.75 \mathrm{MPa}$. The maximum von Mises stress is $61.12 \mathrm{MPa}$. However, these areas are very localized and plastic deformation, if any, will be minimal. The maximum first principal total strain is $2.9 \times 10^{-4}$, which is significantly under the 0.002 strain where yield is reported, suggesting no plastic deformation will actually take place.

Fatigue does not appear to be an issue. Figure 21 shows the total strain range and stress amplitude are well below the allowable values. Fatigue resistance is reduced as temperature increases. The low cycle fatigue data is taken at $56.7^{\circ} \mathrm{C}$, so using that data is conservative. The high cycle fatigue was generated at room temperature.

Based on the results of this analysis, the following conclusions are drawn: (i) the cavity is resistant to plastic deformation; (ii) the cavity is resistant to ductile rupture; (iii) the cavity is resistant to low cycle fatigue; (iv) the cavity is resistant to high cycle fatigue.

\section{CELL FABRICATION AND MEASUREMENTS}

Test cell fabrication was performed through turning and milling operations, and it is shown in Fig. 22. The axially symmetric cell features such as the nose cones and cell floor and inner and outer diameters were all performed on a computer-numeric control $(\mathrm{CNC})$ lathe with polycrystalline

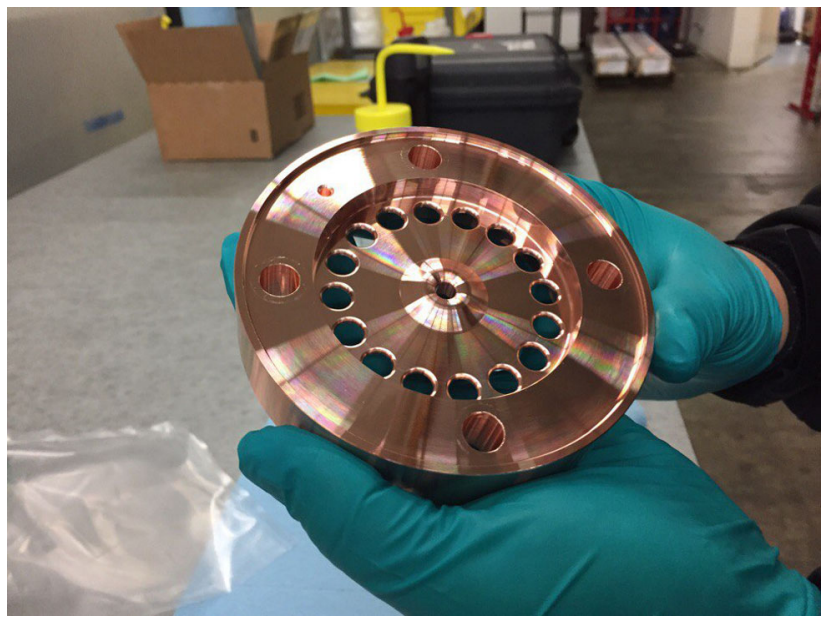

FIG. 22. Finished NHS test cell with all features included.

diamond cutting tools. The coupling, alignment and fastening holes for this cold test prototype were completed on a three-axis $\mathrm{CNC}$ machining center. End plates required for testing were also manufactured with a similar approach.

The frequency sensitivities to critical cell dimensions are summarized in Table IV. The tolerance target of \pm 10 microns, set by the available machining tools, was met with all turned parts, while the positional and form requirements on the coupling holes were kept to 25 microns true position and size. These were validated through coordinate

TABLE IV. Frequency sensitivity of the NHS cell to the critical dimensions, as shown in Fig. 10.

\begin{tabular}{lcc}
\hline \hline Parameter & Dimension & Value \\
\hline$\partial \mathrm{D}_{0} / \partial \mathrm{f}$ & Cell diameter & $-34 \mathrm{kHz} / \mu \mathrm{m}$ \\
$\partial \mathrm{D}_{h} / \partial \mathrm{f}$ & Coupling hole diameter & $-22 \mathrm{kHz} / \mu \mathrm{m}$ \\
$\partial \mathrm{R}_{h} / \partial \mathrm{f}$ & Coupling hole orbit radius & $-2 \mathrm{kHz} / \mu \mathrm{m}$ \\
$\partial \mathrm{x}_{1} / \partial \mathrm{f}$ & Nose cone height & $-64 \mathrm{kHz} / \mu \mathrm{m}$ \\
$\partial \alpha / \partial \mathrm{f}$ & Nose cone opening angle & $-11.8 \mathrm{kHz} / \mu \mathrm{m}$ \\
\hline \hline
\end{tabular}



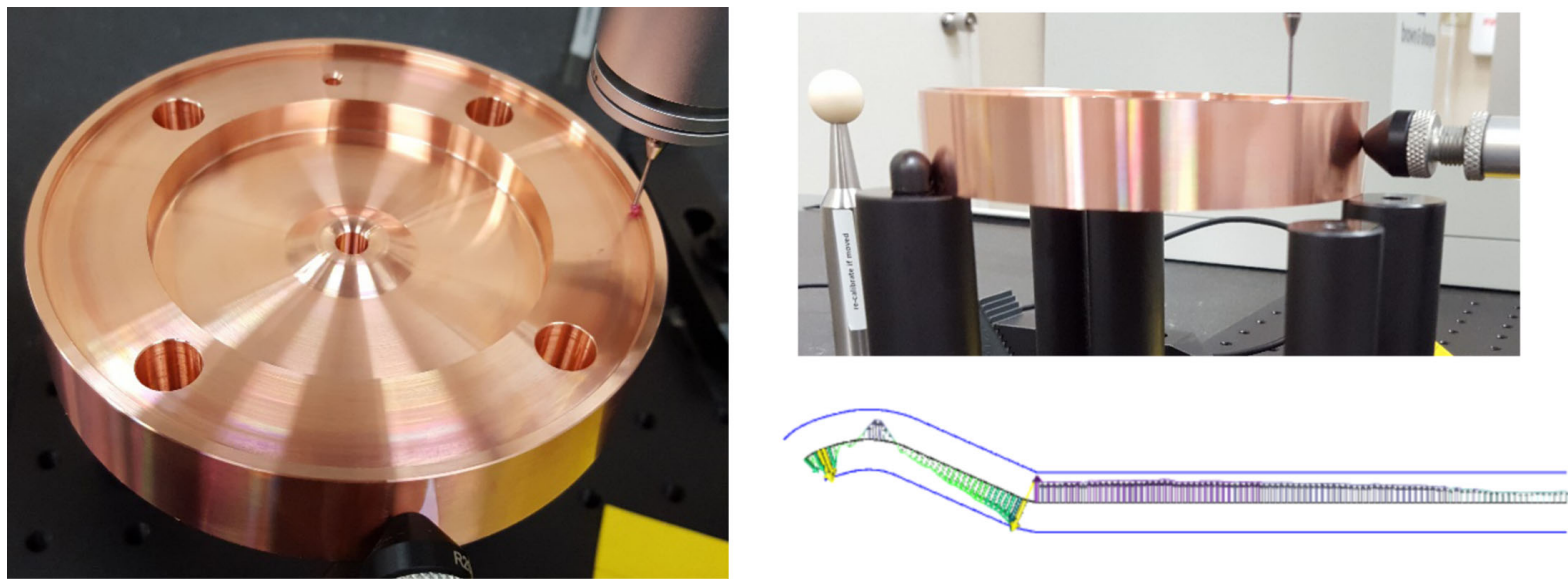

FIG. 23. Turned cell on CMM (left and top right), profile scan taken of the cell showing all dimensions within \pm 10 microns tolerances.
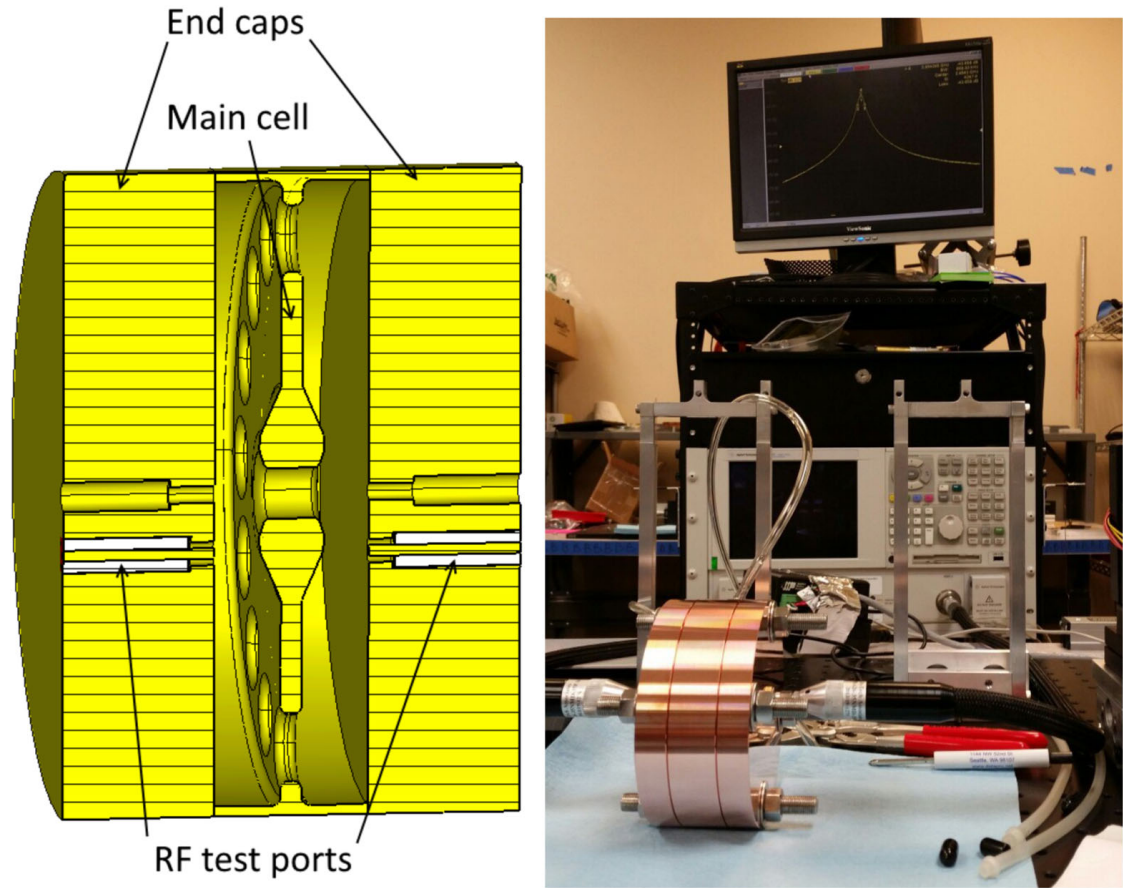

FIG. 24. Single-cell rf test stand design (left) and assembly (right).

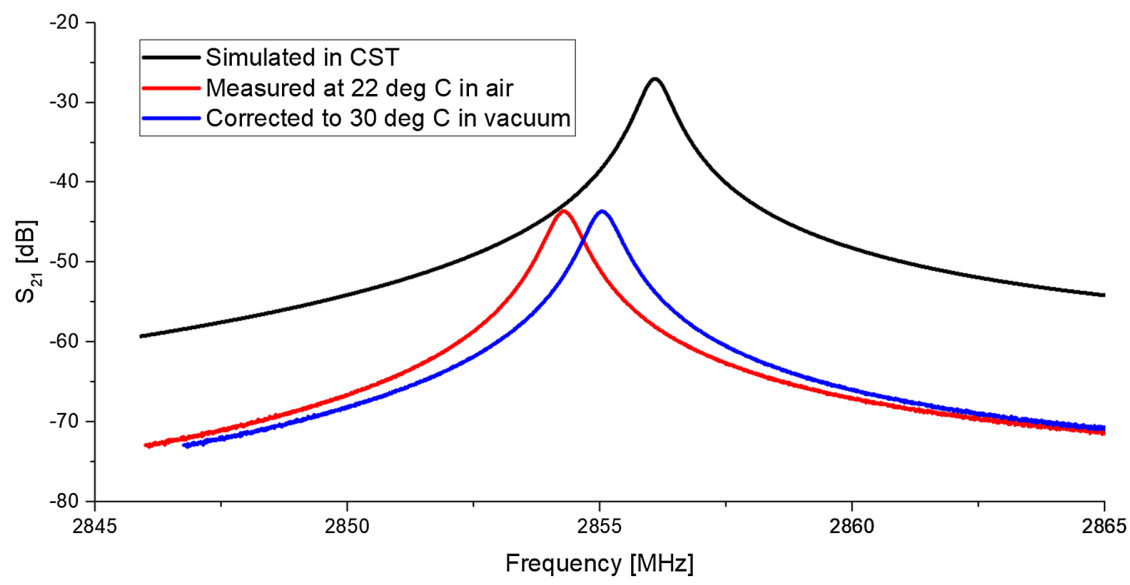

FIG. 25. Simulated (black), measured (red) and corrected (blue) magnitudes of the power transmission $\left(S_{21}\right)$ in a single cell NHS mockup. 
TABLE V. Simulated and measured parameters of the test cell.

\begin{tabular}{lccc}
\hline \hline Parameter & Simulated (vacuum) & Measurements at $22^{\circ} \mathrm{C}$ & Correction to vacuum at $30^{\circ} \mathrm{C}$ \\
\hline$\pi$-mode frequency, $\mathrm{MHz}$ & 2856.11 & 2854.3 & 2855.05 \\
Q-factor of $\pi$-mode & 4700 & 4300 & $\cdots$ \\
\hline \hline
\end{tabular}

measuring machine (CMM) measurements of the test cell as shown in Fig. 23.

We have assembled a test stand and measured the resonant frequency and Q-factor of the cell. The test setup is shown in Fig. 24. The SubMiniature version A probes were adjusted to provide very small coupling $\sim 0.1 \%$ and zero frequency shift. We use the expressions [43] for vacuum-to-room frequency converting:

$$
\begin{aligned}
f_{\text {real }}= & f_{\text {measured }}\left[1+1.7 \times 10^{-5}\left(T_{\text {measured }}-T_{\text {real }}\right)\right] \sqrt{\varepsilon} \\
\varepsilon=1 & +\frac{P_{\text {measured }}}{T_{\text {measured }}} \\
& \times\left[211+\frac{P_{0} H_{\text {measured }}}{P_{\text {measured }}}\left(\frac{10160}{T_{\text {measured }}}-0.294\right)\right] \times 10^{-6}
\end{aligned}
$$

$$
P_{0}=10^{\left(7.45_{\left.\frac{T_{\text {measured }}-273}{T_{\text {masured }}-383}+0.656\right)}\right.} .
$$

Here $T_{\text {measured }}=22^{\circ} \mathrm{C}, \quad H_{\text {measured }}=40 \%, \quad P_{\text {measured }}=$ 762 Torr are measured values. This resulted in a $+0.53 \mathrm{MHz}$ frequency shift between vacuum and room conditions. CST-simulated and measured $\mathrm{S}_{21}$-curves with corrections are presented in Fig. 25, and the comparison of measured and simulated parameters is presented in Table V. The measured $\mathrm{S}_{21}$-curve demonstrates a $\pi$-mode resonance at $2855.05 \mathrm{MHz}$ under vacuum. The frequency shift is compatible with the errors in the dimensions due to the tolerances. The Q-factor of the test cell is lower than the Q-factor of the realistic cell (Table III) due to additional losses in the end caps and operation in $\pi$-mode. The cells for the linac assembly must have mechanical tuners providing at least a $\pm 2.5 \mathrm{MHz}$ tuning range to cover the possible frequency deviations caused by \pm 10 microns machining accuracy with a sufficient margin. The achieved machining tolerances of the cell are within the expected values. We will also consider operation at a temperature of $30{ }^{\circ} \mathrm{C}$ since it tends to result in the frequencies being closer to the designed values as well as increasing the copper yield stress.

\section{SUMMARY}

In this paper, we have described the novel concept of a high gradient accelerating structure, operating at the first negative spatial harmonic, and suitable for the acceleration of low $\beta(<0.4)$ ions that is planned to be used as a starting high gradient section in the Advanced Compact Carbon Ion Linac (ACCIL). We have performed electromagnetic optimization of the $\beta=0.3 \mathrm{NHS}$ cell to achieve $50 \mathrm{MV} / \mathrm{m}$ gradient with $160 \mathrm{MV} / \mathrm{m}$ peak electric field, and shunt impedance of $31.7 \mathrm{M} \Omega / \mathrm{m}$, which is $50 \%$ higher than that of the cell operating at the fundamental spatial harmonic. Such an advancement became possible thanks to the larger gap between the irises and the possibility to fit the noses. Pulsed heating and modified Poynting vector-two other important parameters for RFBD-were kept below their known limits. We have verified the concept in simple beam dynamics simulations and demonstrated the required energy gain per section, corresponding to $50 \mathrm{MV} / \mathrm{m}$ gradient. We have performed thorough thermal and structural analysis to verify the analytical estimations for the pulsed heating and ensure the mechanical stability of the structure. A test cell of the optimized geometry was fabricated and measured, demonstrating the results within the expected range.

The proposed acceleration with higher spatial harmonics has enabled the development of a novel high-gradient accelerating structure design for protons and carbon ions. The prototype structure being developed will become the enabling technology for compact hadron therapy linacs. The successful completion of this project resolves the most acute technical risk in the realization of the compact carbon ion linac ACCIL and becomes a major milestone towards demonstrating the technical feasibility of the entire linac-based cancer therapy system. It will also lead to improved availability and affordability of highly promising ion radiation therapy for a wider range of tumors. The goal of such therapy system is to save patients' lives, improve their quality of life, and offer hope to those whose poor prognosis defies the conventional standards of care.

Besides the direct application to ion therapy, the developed structure can also be applied to future high-gradient normal conducting ion linacs and high energy physics machines, such as compact hadron collider. It can also be utilized in industrial systems, including linacs for ion implantation, material science, and laboratory scale research instrumentation.

\section{ACKNOWLEDGMENTS}

This work was supported by the U.S. Department of Energy, Office of High Energy Physics, under STTR Grant No. DE-SC0015717 and Accelerator Stewardship Grant, 
Proposal No. 0000219678. The authors would also like to thank Valery Dolgashev from SLAC, as well as Stephano Benedetti and Alexej Grudiev from CERN for important discussions about high gradient physics.

[1] M. Durante and H. Paganetti, Nuclear physics in particle therapy: A review, Rep. Prog. Phys. 79, 096702 (2016).

[2] Office of high energy physics, Accelerator R\&D task force report, 2012, pp. 32-37, http://www.acceleratorsamerica .org/report/accelerator_task_force_report.pdf.

[3] Progress in Ultrafast Intense Laser Science XII, edited by K. Yamanouchi, L. Roso, R. Li, D. Mathur, and D. Normand (Springer International Publishing Switzerland, 2015).

[4] K. Peach, P. Wilson, and B. Jones, Accelerator science in medical physics, Br. J. Radiol. 84, S004 (2011).

[5] C. Hu, Catching the proton wave, The National Association for Proton Therapy, http://www.proton-therapy.org/ hhnarticle.htm.

[6] N. Maliwal, Radiotherapy: Technologies and global markets, BCC research, 2015.

[7] Hadron therapy patient statistics: Particle therapy cooperative group, 2013, http://www.ptcog.ch/archive/patient_ statistics/Patientstatistics-updateMar2013.pdf.

[8] U. Amaldi and G. Kraft, Radiotherapy with beams of carbon ions, Rep. Prog. Phys. 68, 8 (2005).

[9] A. J. Lomax, Charged particle therapy: The physics of interaction, Cancer J. 15, 285 (2009).

[10] H. Owen, D. Holder, J. Alonso, and R. MacKay, Technologies for delivery of proton and ion beams for radiotherapy, Int. J. Mod. Phys. A 29, 1441002 (2014).

[11] U. Amaldi, M. Crescenti, and R. Zennaro, Ion acceleration system for hadron therapy, U.S. Patent No. 7423278.

[12] S. Benedetti, A. Grudiev, and A. Latina, High gradient linac for proton therapy, Phys. Rev. Accel. Beams 20, 040101 (2017).

[13] S. Benedetti, A. Degiovanni, A. Grudiev, W. Wuensch, and U. Amaldi, rf design of a novel S-band backward travelling wave linac for proton therapy, in Proceedings of 27th Linear Accelerator Conference, Geneva, Switzerland, 2014 (JACoW, Geneva, 2014), p. 992.

[14] P. N. Ostroumov, A. Goel, B. Mustapha, A. Nassiri, A. S. Plastun, L. Faillace, S. V. Kutsaev, and E. A. Savin, Compact carbon ion linac, in Proceedings of 2016 North American Particle Accelerator Conference, Chicago, IL, USA, 2016 (JACoW, Chicago, 2017) p. 61.

[15] C. J. Karzmark, C.S. Nunan, and E. Tanabe, Medical Electron Accelerators (McGraw-Hill Inc., New York, 1993).

[16] J. B. Rosenzweig, A. Cahill, V. Dolgashev, C. Emma, A. Fukusawa, R. Li, C. Limborg, J. Maxson, P. Musumeci, A. Nause, R. Pakter, R. Pompili, R. Roussel, B. Spataro, and $\mathrm{S}$. Tantawi, Next generation high brightness electron beams from ultrahigh field cryogenic radiofrequency photocathode sources, arXiv:1603.01657.

[17] P. M. Lapostolle and A. Septier, Linear Accelerators (North-Holland, Amsterdam, 1970).

[18] U. Amaldi, P. Berra, K. Crandall, D. Toet, M. Weiss, R. Zennaro, E. Rosso, B. Szeless, M. Vretenar, C. Cicardi,
C. De Martinis, D. Giove, D. Davino, M. R. Masullo, and V. Vaccaro, LIBO a linac-booster for proton therapy: Construction and test of a prototype, Nucl. Instrum. Methods Phys. Res., Sect. A 521, 512 (2004).

[19] S. V. Andrés, U. Amaldi, and A. Faus-Golfe, CABOTO, a high-gradient linac for hadrontherapy, Journal of Radiation Research 54, i155 (2013).

[20] U. Amaldi, S. Braccini, and P. Pugionni, High frequency linacs for hadronotherapy, Rev. Accel. Sci. Techol. 02, 111 (2009).

[21] L. Picardi, C. Cianfarani, G. Messina, G. L. Orlandi, C. Ronsivalle, E. Cisbani, and S. Frullani, High power rf tests of the first module of the top linac SCDTL structure, in Proceedings of the 10th European Particle Accelerator Conference, Edinburgh, Scotland, 2006 (EPS-AG, Edinburgh, Scotland, 2006).

[22] A. Garonna, U. Amaldi, V. Bencini, D. Bergesio, D. C. Perez, C. Cuccagna, E. Felcini, P. Riboni, and M. Varasteh, Advanced linac solutions for hadrontherapy, at the Workshop on Innovative Delivery Systems in Particle Therapy, Torino, 2017, https://agenda.infn.it/getFile.py/ access? contribId $=17 \&$ session $I d=4 \&$ res $I d=0 \&$ materialId $=$ slides\&confId=12108.

[23] A. Plastun, B. Mustapha, A. Nassiri, P. N. Ostroumov, L. Faillace, S. V. Kutsaev, and E. A. Savin, Beam dynamics studies for compact carbon ion linac for therapy, Proceedings of 28th Linear Accelerator Conference, East Lansing, MI, USA, 2016 (JACoW, East Lansing, 2017), p. 946.

[24] A. Grudiev, S. Calatroni, and W. Wuensch, New local field quantity describing the high gradient limit of accelerating structures, Phys. Rev. ST Accel. Beams 12, 102001 (2009).

[25] V. A. Dolgashev, S. Tantawi, Y. Higashi, and B. Spataro, Geometric dependence of radio-frequency breakdown in normal conducting accelerating structures, Appl. Phys. Lett. 97, 171501 (2010).

[26] G. Dome, CERN Internal Report No. ISR/300/LI/70, 1970.

[27] T. Wangler, RF Linear Accelerators, 2nd ed. (Wiley-VCH, Weinheim, Germany, 2008).

[28] V. N. Aseev, P. N. Ostroumov, E. S. Lessner, and B. Mustapha, TRACK: The new beam dynamics code, in Proceedings of the 21st Particle Accelerator Conference, Knoxville, TN, 2005 (IEEE, Piscataway, NJ, 2005), p. 2053.

[29] I. M. Kapchinskiy, Theory of Linear Resonance Accelerators (Harwood Academic, New York, 1985).

[30] C. Bieth, S. Kantas, P. Sortais, D. Kanjilal, and G. Rodrigues, Recent development in ECR sources, Nukleonika 48, S93 (2003).

[31] V.A. Dolgashev, High magnetic fields in couplers of $\mathrm{x}$-band accelerating structures, in Proceedings of the 2003 Particle Accelerator Conference, Portland, OR (IEEE, New York, 2003).

[32] L. Faillace, R. Agustsson, P. Frigola, A. Murokh, V. Dolgashev, J. Rosenzweig, and V. Yakimenko, Fabrication and initial tests of an ultrahigh gradient compact S-band (HGS) accelerating structure, in Proceedings of the $3 \mathrm{rd}$ International Particle Accelerator Conference, New Orleans, LA, 2012 (IEEE, Piscataway, NJ, 2012), p. 3392.

[33] M. Vretenar, CERN Yellow Report No. CERN-2011-007, pp. 319-340. 
[34] S. Kutsaev, R. Agustsson, L. Faillace, A. Goel, B. Mustapha, A. Nassiri, P. Ostroumov, A. Plastun, and E. Savin, High gradient accelerating structures for carbon therapy linac, in Proceedings of 28th Linear Accelerator Conference, East Lansing, MI, USA, 2016 (JACoW, East Lansing, 2017), p. 851.

[35] N. P. Sobenin and B. V. Zverev, Electrodynamic Characteristics of Accelerating Cavities (CRC Press, London, 1999), p. 288.

[36] D. Tronc, Traveling wave acceleration of protons, Nucl. Instrum. Methods Phys. Res., Sect. A 327, 253 (1993).

[37] G. A. Loew, Report No. SLAC-PUB-3221, 1983.

[38] G. Li, B. G. Thomas, and J. F. Stubbins, Metall. Mater. Trans. A 31, 2491 (2000).

[39] P. C. Bolur, A Guide to Injection Molding of Plastics, 3rd ed., http://www.pitfallsinmolding.com.
[40] S Kakaç et al., Handbook of Single Phase Convective Heat Transfer (John Wiley and Sons, New York, 1987).

[41] J. Esposito and R. Zabora, Thrust Chamber Life Prediction, Volume I, Mechanical and Physical Properties of High Performance Rocket Nozzle Materials, NASA CR 124806 (Boeing Aerospace Co., Seattle, WA, 1975).

[42] J. E. Jensen, W. A. Tuttle, R. B. Stewart, H. Brechna, and A. G. Prodell, Selected Cryogenic Data Notebook, Volume I, BNL 10200-R (Brookhaven National Laboratory, Upton, NY, 1980).

[43] T. Khabiboulline, V. Puntus, M. Dohlus, N. Holtkamp, G. Kreps, S. Ivanov, and K. Jin, A new tuning method for traveling wave structures, in Proceedings of the Particle Accelerator Conference, Dallas, TX, 1995 (IEEE, New York, 1995). 